\title{
Gas Mass Fractions and the Evolution of Spiral Galaxies
}

\author{
Stacy S. McGaugh \\ Department of Terrestrial Magnetism, Carnegie Institution of Washington \\ and \\ W. J. G. de Blok \\ Kapteyn Astronomical Institute, University of Groningen
}

\begin{abstract}
We show that the gas mass fraction of spiral galaxies is strongly correlated with luminosity and surface brightness. It is not correlated with linear size. Gas fraction varies with luminosity and surface brightness at the same rate, indicating evolution at fixed size.

Dim galaxies are clearly less evolved than bright ones, having consumed only $\sim 1 / 2$ of their gas. This resolves the gas consumption paradox, since there exist many galaxies with large gas reservoirs. These gas rich galaxies must have formed the bulk of their stellar populations in the last half of a Hubble time. The existence of such immature galaxies at $z=0$ indicates that either galaxy formation is a lengthy or even ongoing process, or the onset of significant star formation can be delayed for arbitrary periods in tenuous gas disks.
\end{abstract}

Subject headings: galaxies: evolution — galaxies: formation — galaxies: fundamental parameters — galaxies: photometry — galaxies: spiral — galaxies: structure

Accepted for Publication in the Astrophysical Journal 


\section{Introduction}

The evolutionary history of galaxies can be inferred in a variety of ways. One approach is to utilize the extensive knowledge of the evolution of stars gleaned from the HR diagram to construct model galaxies (e.g., Tinsley 1968; Bruzual \& Charlot 1993). More recently, it has become possible to infer related information in a statistical way from deep redshift surveys (e.g., Lilly et al. 1995; Ellis et al. 1996). Piecing the various lines of evidence together is not trivial, and rather different pictures can emerge from equally valid approaches.

It is essential that the question be well posed. What is meant by galaxy evolution? We generally imagine that galaxies form out of the gas that emerges from the hot big bang. Regardless of the details of galaxy formation, the units which we observe as galaxies today have converted some fraction of this gas into stars. The gas mass fraction $f_{g}$ is thus a natural parameter for quantifying the degree of evolution of a galaxy.

This obvious path has not been much pursued for the simple reason that most well observed galaxies have low gas fractions $\left(f_{g} \lesssim 0.2\right)$. Their evolution is well advanced, and the small dynamic range in $f_{g}$ provides no useful constraint on models. Indeed, this leads to a conundrum in that we appear to live at a special time when most disks are near to exhausting their gas reservoirs (e.g., Roberts 1963; Kennicutt et al. 1994).

However, those objects which are well studied need not be representative. It has recently been demonstrated that disk galaxies of low surface brightness are quite common (McGaugh et al. 1995a, de Jong 1996, McGaugh 1996a; Impey et al. 1996). These galaxies are generally gas rich (Schombert et al. 1992; de Blok et al. 1996), and $M_{\mathrm{HI}} / L$ increases systematically with decreasing surface brightness (Fig. 1). This ratio depends only on the relative amounts of atomic hydrogen and star light, and must be an evolutionary effect. Attempting to understand this evolution and its strong dependence on surface brightness is the subject of this paper.

We emphasize that our discussion is restricted to disk galaxies, primarily spirals. We do not address Elliptical or dwarf Spheroidal galaxies, though we do thoroughly examine relevant selection effects. When required, distance dependent quantities assume $H_{0}=$ $75 \mathrm{~km} \mathrm{~s}^{-1} \mathrm{Mpc}^{-1}$.

\section{The Data}

The data consist of optical and $21 \mathrm{~cm}$ fluxes. Data are selected to provide as large as possible a database for investigating the relation between the gas content and optical properties of spiral galaxies, particularly surface brightness. A prerequisite is that each of the quantities of interest are actually measured. Surface brightness, by which we mean the central surface brightness of the disk determined from extrapolation to $R=0$ of exponential fits to the observed light profile, is not commonly measured and is therefore the most limiting factor. Of studies which specifically measure surface brightness in the appropriate fashion, HI measurements are not always available, further limiting the available data. It would be desirable to also have $\mathrm{CO}$ measurements as a tracer of the molecular gas, but there are essentially no published data which meet this additional criterion, and low surface brightness galaxies have yet to be detected in CO at all (Schombert et al. 1990). Instead, we will use the gas phase-morphological type relation of Young \& Knezek (1989) molecular gas content. A further piece of information required for estimating the stellar mass is either an $I$ magnitude or a $B-V$ color (see §3.1). In sum, we need to know $M_{B}, \mu_{0}, h, M_{\mathrm{HI}}$, the morphological type $\mathcal{T}$, and $B-V$ or $I$.

The large data sets which meet these requirements are those of Romanishin et al. (1982; 1983), McGaugh \& Bothun (1994), de Jong \& van der Kruit (1994), de Jong $(1995 ; 1996)$, and de Blok et al. $(1995 ; 1996)$. Surface photometry has been performed on all galaxy images to determine the disk parameters $\mu_{0}$ (central surface brightness) and $h$ (scale length), as described in the various sources. Most of the data for higher surface brightnesses $\left(\mu_{0}<23 \mathrm{~B}\right.$ mag $\left.\operatorname{arcsec}^{-2}\right)$ are from de Jong, whereas most of that for $\mu_{0}>23$ are from our own work. An obvious gap visible in Fig. 1(b) at intermediate surface brightnesses between these two data sets is filled in Fig. 1(a) by the data of Romanishin. Where used, data for the special case of Malin 1 are taken from Bothun et al. (1987) and Impey \& Bothun (1989).

No trends with $M_{\mathrm{HI}} / L$ are obvious in any one data set alone. Only upon combining them does the strong relation in Fig. 1 become apparent. Data over this large dynamic range (a factor of 100) in surface brightness have not previously been available. 


\subsection{Selection Effects}

The data have been selected to be as inclusive as possible given the demands placed on information content. We make no claim that these data are complete in any volume limited sense. For our present purposes, this is less important than having data representative of all morphological types, luminosities, etc. This is because we are more interested in the evolution of galaxies as physical systems than in the number density of any given type. Nonetheless, the potential effects of selection upon interpretation warrant further discussion.

The optical properties of the galaxies are plotted in Figs. 2 and 3. These illustrate some important basic facts about spiral galaxies. Disks exist over a large range in luminosity, surface brightness, and size. No particular value is characteristic for any of these; each has some distribution (McGaugh 1996a; de Jong 1996). Lower surface brightness disks tend to be bluer (Fig. 3), not redder as expected if they have fading evolutionary histories (McGaugh \& Bothun et al. 1994). The lack of red low surface brightness galaxies may itself be a selection effect (McGaugh et al. 1996a), but one still wishes to understand why the ones we do know about are so very blue.

Flux limited surveys are most efficient at identifying the brightest $\left(L^{*}\right)$ and highest contrast $\left(\mu_{0}{ }^{*}\right)$ galaxies that exist in the intrinsic distributions in a given passband. As a direct consequence, galaxy catalogs are numerically dominated by such objects far out of proportion to their actual numbers. Samples selected for detailed study from such catalogs naturally tend to concentrate on these most prominent galaxies. We have avoided this by augmenting such data with our own data for low surface brightness galaxies. As a result, the combined data provide as comprehensive a view of spiral galaxies in the field as is currently possible, spanning the full range of disk morphologies from Sa to $\mathrm{Sm} / \mathrm{Im}$ with $\mu_{0}<25$ and $h>1 \mathrm{kpc}$.

Though this procedure adds sorely needed weight to late types and low surface brightnesses, by no means does it guarantee a homogeneous sample. One indication of this is present in Fig. 2 as an apparent lack of low luminosity, high surface brightness galaxies with $M_{B}>-18$ and $\mu_{0}<23$. This gap is not apparent when $\mu_{0}$ is plotted against $h$ (McGaugh 1996b), and does not necessarily indicate a real lack of such galaxies. In effect, the sample of de Jong contains only bright $\left(M_{B}<-18\right)$ galaxies while our own contains only ones of low surface brightness $\left(\mu_{0}>23\right)$. The excluded region could well be populated, but no data which meet all our information requirements exist in this region of parameter space.

To test whether this can have a serious impact on our results, we perform the analysis described below for both the entire sample, and a subsample limited to $M_{B}<-18$. Though the lever arm for fainter galaxies is of course weaker, no serious differences occur among gas related parameters. The reason for this is simple: the galaxies are optically selected. Conclusions about the relation between optical parameters relevant to selection $\left(M_{B}, \mu_{0}\right.$, and $\left.h\right)$ are difficult to draw in the absence of a rigorously complete sample. However, the investigation into the evolution of galaxies is not much hindered because a wide range of these parameters are at least represented. Moreover, the initial selection of the galaxies is independent of $M_{H I}$. These data tell us quite a bit about the evolution of the galaxy types we do know about, and delimit what may still be missing.

\section{Gas Mass Fractions}

The baryonic gas mass fraction is

$$
f_{g}=\frac{M_{g}}{M_{g}+M_{*}}
$$

where $M_{g}$ is the total mass in the form of gas, and $M_{*}$ that in stars. To relate these to the observations, we need $M_{*}=\Upsilon_{*} L$ and $M_{g}=\eta M_{\mathrm{HI}}$. The parameter $\Upsilon_{*}$ is the mass to light ratio of the stellar population, while $\eta$ plays an analogous role for the gas. It accounts both for helium and any mass in metals and for gas phases other than atomic.

With these definitions, it is straightforward to obtain

$$
f_{g}=\left(1+\frac{\Upsilon_{*} L}{\eta M_{\mathrm{HI}}}\right)^{-1}
$$

The flux ratio $M_{\mathrm{HI}} / L$ is distance independent and has small errors in most cases. However, the conversion factors $\Upsilon_{*}$ and $\eta$ must be estimated from other information.

\subsection{Stellar Population Mass to Light Ratios}

We wish to use the observable star light as an indicator of the stellar mass. Our stellar mass to light ratio $\Upsilon_{*}$ includes all mass in stars living or dead since 
we wish to distinguish between the gaseous and stellar mass reservoirs. The value of $\Upsilon_{*}$ can be estimated from population synthesis models, but these are highly uncertain and depend on many factors like the IMF, star formation history, metallicity, gas recycling, the poorly known behavior of some late stages of stellar evolution, etc. Even worse for our purposes, it depends iteratively on the quantity of interest, $f_{g}$. Though such models are still very useful, it is of obvious interest to have independent means of constraining $\Upsilon_{*}$.

We use the dynamical constraints imposed by observations of the vertical stellar velocity dispersion (Bottema 1993), as generalized by de Blok \& McGaugh (1997). This gives

$$
\Upsilon_{*}=1.936 \times 10^{0.4(B-V)}-1.943
$$

in the $B$-band. This expression is derived from the disk Tully-Fisher relation with normalization imposed by the velocity dispersion observations. There is some uncertainty in the absolute normalization, but considerably less than in estimates lacking the boundary condition imposed by dynamical measurements. The color term has a residual dependence on population synthesis which goes in the expected sense that redder galaxies have higher $\Upsilon_{*}$. This reproduces the observed trend well (Fig. 4) even though one does not expect $B-V$ to be a perfect indicator of the variation of $\Upsilon_{*}$ with the many underlying variables.

The most important aspect of equation (3) is the normalization to dynamical constraints which give the most reliable estimate of the mass contained in stars. Without this, it is always possible to contrive models in which the stars contain all or none of the baryonic mass, and $f_{g}$ remains highly uncertain. With it, $f_{g}$ is reasonably well determined.

The numbers returned by equation (3) are consistent both with stellar population models (e.g., van den Hoek et al. 1996) and rotation curve analyses (Sanders 1996; de Blok \& McGaugh 1997) which are independent of the velocity dispersion measurements. As a further independent check of our results, we will also compute $f_{g}$ from a purely population synthesis estimate of $\Upsilon_{*}^{I}$ in the $I$-band. The $I$-band is thought to be the most robust indicator of stellar mass (Worthey 1994), though one still does not expect a universal value for all populations. The models of Bruzual \& Charlot (1993) for a galaxy with constant star formation suggest a value of $\Upsilon_{*}^{I} \approx 1.2$ over a wide range of ages, with fairly modest variation.
Though both the absolute normalization and the universal applicability of this value is uncertain, the use of this fixed number in the $I$-band gives indistinguishable results from those of equation (3) in the $B$-band (Fig. 5).

\subsection{Gas Phases}

For the gas, $\eta$ must be corrected for both the hydrogen mass fraction $X$, and the phases of gas other than atomic. We assume a solar hydrogen mass fraction, giving $\eta=X_{\odot}^{-1}=1.4$. Though not universally correct, most of this is due to primordial helium. Variations in helium and metal content result in deviations from this value of less than $10 \%$. This is a very small effect compared to that of other gas phases. Ionized gas in H II regions and hotter plasma is of negligible mass in spiral galaxies, but not so molecular gas. Hence,

$$
\eta=1.4\left[1+\frac{M\left(\mathrm{H}_{2}\right)}{M(\mathrm{HI})}\right] .
$$

The ratio of molecular to atomic gas varies systematically with Hubble type (Young \& Knezek 1989). We parameterize their mean relation by

$$
\frac{M\left(\mathrm{H}_{2}\right)}{M(\mathrm{HI})}=3.7-0.8 \mathcal{T}+0.043 \mathcal{T}^{2}
$$

(Fig. 6). For very late types, we have forced the fit to approach $M\left(\mathrm{H}_{2}\right) / M(\mathrm{HI}) \rightarrow 0$ as $\mathcal{T} \rightarrow 10$. Equation (5) gives a good fit to the trend of the data of Young \& Knezek (1989) except perhaps for very early types, but there are few galaxies in the sample with $\mathcal{T}=1$. Of greater concern is the large amount of real scatter at each type $-\mathcal{T}$ is by no means a perfect indicator of $M\left(\mathrm{H}_{2}\right) / M(\mathrm{HI})$, so equation (5) is only a statistical estimator of the relative molecular gas content.

The normalization of equation (5) depends on the $\mathrm{CO}$ to $\mathrm{H}_{2}$ conversion factor, the precise value and universality of which is a matter of much debate. The conversion factor appears to be metallicity dependent (Wilson 1995), and later types are generally more metal poor than early types. If one were to correct the noisy correlation of $M\left(\mathrm{H}_{2}\right) / M(\mathrm{HI})$ with $\mathcal{T}$ for the noisy correlation of $Z$ with $\mathcal{T}$, it would have only a modest effect on equation (5) since the inferred variation in the conversion factor with metallicity is fairly small compared to the range in Fig. 6. Little will change because early types are approximately solar metallicity and will receive no correction from the 
canonical value of the conversion factor. The latest types are roughly $0.1 Z_{\odot}$ (McGaugh 1994a), corresponding to a factor of 5 increase in the conversion factor. However, $M\left(\mathrm{H}_{2}\right) / M(\mathrm{HI})$ is inferred to be very small in late types, and a five fold increase in a small number still results in a very small correction to the final $f_{g}$. Rather than convolve together all these uncertainties, we simply use equation (5), realizing that this is probably the greatest uncertainty in the process.

The uncertainty in the correction for molecular gas will have little impact on our final results. The correction is only important in early types, which are relatively gas poor $f_{g} \lesssim 0.3$. It is the late types which are inferred to be gas rich, on the basis of the HI alone. If we are missing enormous amounts of molecular gas in late types it will only make yet more gas rich galaxies which are already inferred to be very gas rich. Hence our qualitative results are very robust, even if the quantitative details are subject to improvement in the CO data. Moreover, it is only the ratio $\Upsilon_{*} / \eta$ that matters to our analysis (equation 2). This ratio does not vary much, and is not correlated with surface brightness. In order to affect our results, this ratio would have to vary systematically with surface brightness by an order of magnitude in just the right way to offset the strong observed trend in $M_{H I} / L$. Not only does this seem unlikely, it simply can not happen. The gas fractions of low surface brightness galaxies can not be lowered by large $\Upsilon_{*}$ as these are not allowed by the rotation curve data (de Blok \& McGaugh 1996), nor can $\eta$ be raised for high surface brightness galaxies where $\mathrm{CO}$ is readily detected. Hence the large observed variation in $M_{H I} / L$ must correspond to one in $f_{g}$.

The role of molecular and atomic gas: It is generally thought that star formation occurs only in molecular gas. However, it appears that there is very little and perhaps no molecular gas in the lowest surface brightness galaxies (Schombert et al. 1990). We should therefore consider the possibility that stars form directly in from atomic gas. It is not obvious that this is necessary, though. The star formation rates are low, and a very modest amount of molecular gas might exist as an intermediary step. Indeed, one could argue that the lack of molecular gas inhibits star formation and keeps the surface brightness low. While these are interesting possibilities, they do not matter to our present analysis, which is concerned only with the global supply of gas and not the details of its local consumption. In this context, it is perhaps best to think of the $\mathrm{HI}$ as a reservoir for future star formation, regardless of whether molecular gas is a mandatory intermediate step. Indeed, gradual radial infall from the extended HI disks of spirals may be the source of the inferred accretion of unprocessed material since there is no evidence for substantial HI reservoirs in spherical halos (Bothun 1985).

\section{The Relations}

The gas mass fractions computed as described above are tabulated in Table 1 together with the input data. In general there is close correspondence between $f_{g}$ computed from $B$-band and $I$-band data. This is encouraging since independent methods are used to estimate $\Upsilon_{*}$ which is the more important factor in equation 2. Variation in the two different determinations of $f_{g}$ give some idea of the uncertainties therein.

In Fig. 7 we plot the gas fractions against the $B$ band properties of the galaxies. A strong relation between gas fraction and total luminosity, disk surface brightness, and color is apparent. The same relations with luminosity and surface brightness are also apparent in the $I$-band data (Fig. 8). There is no relation at all with the size of the optical disk. Fits to the $B$-band data give

$$
\begin{array}{r}
f_{g}=0.12\left(M_{B}+23\right) \\
f_{g}=0.12\left(\mu_{0}-19\right) \\
f_{g}=-1.4[(B-V)-0.95]
\end{array}
$$

where the uncertainty in the slope is \pm 0.07 for $M_{B}$, \pm 0.04 for $\mu_{0}$, and \pm 0.5 for color. These relations are of course subject to modest variation with improvements in the relations governing $\Upsilon_{*}$ and $\eta$, but it is clear that dimmer galaxies are systematically more gas rich. The slopes of the $f_{g}-M_{B}$ and $-\mu_{0}$ relations are indistinguishable.

An important consequence of the systematic increase of gas fraction with surface brightness is the end of the gas consumption paradox. Since roughly equal numbers of galaxies exist at each surface brightness, there are many galaxies which still retain substantial gas reservoirs (see McGaugh 1996a for the distribution functions). Perhaps it is not surprising that the galaxies which are the most prominent optically are those which have converted most of their gas into stars. 


\subsection{Correlations}

Correlation coefficients from a principle component analysis of the $B$-band data are given in Table 2 . When reading this table, be sure to remember the backwards convention in the definition of magnitudes. This affects the signs of the correlation coefficients: $\mathcal{R}=-0.59$ between $M_{B}$ and $h$ means that brighter galaxies tend to be bigger, not smaller.

First note that $f_{g}$ is well correlated with $M_{H I} / L$, from which it is derived. This was not entirely a foregone conclusion, since the relations for $\Upsilon_{*}$ and $\eta$ could conceivably have offset this. The use of these relations introduces noise which reduces the correlation coefficient from unity.

Amongst independent variables, the strongest correlation is between $M_{H I} / L$ and $\mu_{0}$. This relation is more significant than either of the well known relations between $M_{H I} / L$ and $M_{B}$ (Rao \& Briggs 1993; Salpeter \& Hoffman 1996) or $M_{H I} / L$ and $\mathcal{T}$ (Roberts 1963), though these are also obvious in Table 2. This strong $M_{H I} / L-\mu_{0}$ relation translates into a strong one between $f_{g}$ and $\mu_{0}$. The central surface brightness of a galaxy is a good indicator of its cumulative gas consumption and hence its evolutionary state.

The gas fraction is also correlated with $M_{B}$ and $B-V$. Though both are used in the calculation of $f_{g}$, this correlation is not an artifact. It stems from the basic fact that there is relatively more $21 \mathrm{~cm} \mathrm{lu}$ minosity in spirals of low optical luminosity, which also tend to be bluer. The relation with color goes in the sense that blue galaxies are more gas rich, as one would expect. Moreover, the same result is obtained when color is not used in the calculation of $f_{g}$ with the $I$-band data.

The data appear to occupy a fairly narrow structure in $f_{g}-\mu_{0}$-color- $M_{B}$ space. In analogy to the Fundamental Plane for ellipticals, this may indicate the existence of an "Evolutionary Hypersurface" constraining the evolution of spirals. However, this is violated by the extreme galaxy Malin 1 which is exceedingly gas rich for its luminosity. A more fundamental plane may be that in $f_{g}-\mu_{0}$-color space, but this too may be modified by further selection effects (see below).

The gas fraction is not well correlated with size. The evolutionary rate of spiral galaxies is apparently scale free. Neither is $f_{g}$ correlated with Hubble type, in spite of the $M_{H I} / L-\mathcal{T}$ relation. This is because the dominant gas phase changes rapidly with Hubble type (Young \& Knezek 1989), offsetting in the total gas fraction the trend apparent in the atomic phase alone.

Among optical parameters, Hubble type depends most on $M_{B}$ and $\mu_{0}$. Though it is not surprising that these are relevant to the morphological appearance of a galaxy, $\mathcal{T}$ does not provide a clean measure of any physical parameter (Naim et al. 1995; McGaugh 1995b; de Jong 1995). Type is not well correlated with size, though there is some tendency for earlier types to be redder.

The linear size of a disk galaxy is not well correlated with anything except absolute magnitude. Absolute magnitude is appears to be reasonably well correlated with surface brightness in the obvious sense that higher surface brightness galaxies are brighter. However, this may be illusory, being the relation most subject to selection effects. In particular, essentially all the low luminosity galaxies are low surface brightness (recall Fig. 2). This may or may not be representative of reality. If we restrict the sample to objects brighter than -18 where the $M_{B}-\mu_{0}$ plane is more completely covered, this correlation coefficient drops to $\mathcal{R}=0.34$. That between $\mu_{0}$ and $h$ increases to $\mathcal{R}=0.44$ - bigger galaxies tend to be lower surface brightness. These are the only significant changes in the correlation matrix when the sample is restricted to bright galaxies.

Optical galaxy selection depends jointly on $M_{B}$, $\mu_{0}$, and $h$ (Disney \& Phillipps 1983; McGaugh et al. 1995a), so correlations between these variables are very sensitive to sample selection and must be treated with caution. Correlations with other variables are not as directly subject to selection effects, and can sensibly be analyzed as long as representative data is available over a reasonable dynamic range. In particular, correlations involving $f_{g}$ appear to be robust.

\subsection{Further Selection Effects}

We should nevertheless consider whether the relations derived above are affected by selection effects which in the past helped conceal them. We have addressed the problem of isophotal selection, to the extent currently possible, by specifically targeting low surface brightness galaxies. This increases the dynamic range available in $\mu_{0}$, but it is obvious that such effects remain from the truncation of the data as $\mu_{0} \rightarrow 25$. The galaxies studied here are selected optically, but of course a galaxy must have detected 
HI to perform this analysis. The lack of low gas fraction, low surface brightness galaxies could therefore be a further selection effect.

The relations appear continuous across samples, which would seem to argue against strong selection effects in this sense. The $21 \mathrm{~cm}$ detection rate is very high $(80 \%)$ for low surface brightness galaxies in the Schombert et al. (1992) catalog, so we do not seem to be missing a significant number of these predominantly spiral galaxies. However, the fact that these galaxies are very blue (Fig. 3) may itself be a selection effect, since they were selected on blue sensitive plates (see McGaugh 1996a). For this to be important to Fig. 7, a large additional population of gas poor red low surface brightness galaxies would also have to exist.

Other types of galaxies (e.g., dE and dSph) are certainly known to exist which have low surface brightnesses and low gas fractions. These particular types bear no apparent relation to the larger low surface brightness spirals cataloged by Schombert et al. (1992), or to higher surface brightness spirals. Nevertheless, it may well be that the observed relations represent only upper envelopes, and a substantial population of gas poor low surface brightness galaxies remains to be discovered. Indeed, such a population may be demanded by the rapid evolution of the luminosity density (Dalcanton 1993; Lilly et al. 1995 see §6). The lack of gas rich, high surface brightness galaxies is not a selection effect: such objects would be prominent both optically and in HI.

\section{Disk Evolution}

Irrespective of these caveats, clear evolutionary trends exist. The lower surface brightness and lower luminosity spirals that we know about have consumed less of their gas and are less evolved than brighter systems. This result is consistent with other lines of evidence from star formation thresholds (van der Hulst et al. 1993), abundances (McGaugh 1994a; Rönnback \& Bergvall 1995), colors (McGaugh \& Bothun 1994; Rönnback \& Bergvall 1994; Bergvall \& Rönnback 1995; de Blok et al. 1995) and environments (Bothun et al. 1993; Mo et al. 1994), all of which indicate that low surface brightness galaxies evolve slowly, and perhaps form late.

On their own, Figs. 7 and 8 suggest a straightforward picture: disk galaxies form and then evolve at constant size, converting gas into stars and hence changing their luminosity and surface brightness at the same rate. This assures strong $f_{g}-M_{B}$ and $f_{g}-\mu_{0}$ relations with the same slope. There need be no correlation between $M_{B}$ and $\mu_{0}$ since galaxies exist over a range of sizes. Disk galaxy evolution thus seems to proceed in an orderly fashion, without excessive amounts of merging, size evolution, strong stochastic star bursts, or other exotic phenomena.

The evolutionary trajectory of a galaxy in Fig. 7 or 8 depends on the evolution of stars it contains, and the rate at which those stars are made. The star formation rate (SFR) is just the negative of the rate of gas consumption: $\dot{f}_{*}=-\dot{f}_{g}$, at least until gas recycling becomes important at low $f_{g}$. The photometric evolution of stars depends on many factors (e.g., their metallicity) which lead to a variety of complications, but the basic physics is well understood and allows one to estimate the luminosity that will be produced by a given mass of stars. This gives the abscissa. The ordinate follows from the SFR which is traditionally parameterized as some convenient function of time (e.g., Guideroni \& Rocca-Volmerange 1987). For declining SFRs in which most of the star formation occurred well in the past, a commonly assumed form is

$$
\dot{f}_{*}=-\dot{f}_{g}=\tau^{-1} e^{-t / \tau} .
$$

The SFR is normalized to the total baryonic mass of a given galaxy so that $0 \leq f_{*} \leq 1$. The $e$-folding time scale $\tau$ is typically several Gyr as thought appropriate for early type galaxies. For SFRs which are constant (appropriate for late type spirals) or rising (as is perhaps the case for irregular galaxies), a useful form is

$$
\dot{f}_{*}=-\dot{f}_{g}=\frac{x+1}{\tau_{g}}\left(\frac{t}{\tau_{g}}\right)^{x} .
$$

In this case, $\tau_{g}$ is the time to total gas consumption. This is not quite the same as an $e$-folding time scale, but in both cases $\tau^{-1}$ sets the evolutionary rate.

We do not delude ourselves that either of these simple SFRs accurately represent what really occurs in any given galaxy. They do nevertheless provide a very useful way of parameterizing the range of possible average star formation histories. This simple analytic approach does enable one to infer several interesting constraints on the more complicated reality when a large dynamic range in $f_{g}$ is available.

Integration of these SFRs allows us to define several quantities of interest (and also illustrates why these forms are simple and popular). The age of a 
galaxy, $T_{G}$, we will take to mean the time since star formation commenced. This is the obvious working definition of galaxy formation in this context. It does not, of course, specify precisely how a galaxy formed. Star formation might well begin while the collapse of gas is ongoing, or might have begun in separate sub units which subsequently merge into the single entity observed today. It is convenient to imagine the case in which the time scale for such formation events is short compared to the evolutionary time scale $\tau$ so that formation is contemporaneous with the onset of star formation and $T_{G}$ is the true age of the galaxy for all practical purposes. This need not be the case, so one should keep in mind that $T_{G}$ really measures the time since the onset of star formation.

Another interesting quantity is the mean age of the stars, $\left\langle T_{*}\right\rangle$. It is often possible to vary the shape of the SFR so as to arrive at any desired $f_{g}$ with a degenerate set of ages $T_{G}$ and evolutionary rates $\tau$. However, otherwise plausible combinations of these two sometimes have implausible consequences for $\left\langle T_{*}\right\rangle$. These and other quantities and limits of interest are given in Table (3).

Equipped with this formalism, we are nearly ready to interpret the positions of galaxies in Fig. 8. The value of $f_{g}$ comes from stipulating an SFR, but the precise trajectory a galaxy follows in luminosity depends on the details of the stars for which a population model is required. This gives the variation of $\Upsilon_{*}$ with time. The amount of light per unit mass produced by a single generation of stars declines rapidly as luminous, massive stars evolve and die. However, if star formation is ongoing for any period of time, this effect is offset by the birth of new stars. To estimate the interpay of these effects, we need to choose a specific population model. For now, we employ the models of Guideroni \& Rocca-Volmerange (1987), briefly described in Table (4). This particular choice is made for the simple reason that they published the full range of $f_{g}$ for an interesting variety of SFRs. Various improvements in modeling have since been made, but no uniform set of models is readily available over the entire dynamic range we need. Moreover, we are trying here to avoid as much as possible the serious astronomical problem of model dependency; specific models are adopted only to illustrate the various issues. Compared to the dynamic range in $f_{g}$, stellar populations experience fairly mild evolution of $\Upsilon_{*}$ in all plausible models. So while the detailed shape of evolutionary tracks is highly model dependent, the basic gist is not.

For example, Fig. 8 by itself does not represent a direct evolutionary sequence. Galaxies do not start at faint $M$ and $\mu_{0}$ and simply brighten along the observed sequence. The slope of the observed relations is an order of magnitude different from what is expected from evolutionary models for the rate of change of absolute magnitude $(\Delta m)$ with gas fraction $\left(\Delta f_{g}\right)$. Instead of the slope observed in equation (6), $\frac{\Delta m}{\Delta f_{g}}=8$, a very broad range of models evolve slowly in luminosity compared to the rate of gas consumption with $-2<\frac{\Delta m}{\Delta f_{g}}<2$ (Fig. 9). This difference between observed and model slopes requires a systematic variation in the evolutionary rate with surface brightness. Progressively dimmer galaxies either evolve more slowly or are younger, or some combination thereof.

The isochrones of the models of Guideroni \& RoccaVolmerange (1987) are roughly parallel to the observed $\frac{\Delta m}{\Delta f_{g}}$ slope for ages $T_{G}>10 \mathrm{Gyr}$. This is achieved by systematically changing the gas consumption time scale with Hubble type (Table 4). Since very little of these data were available when the models were constructed, this is a success of the standard picture of galaxy evolution the models represent. On the other hand, the correlation between $M_{\mathrm{HI}} / L$ and type was known, and the models were constructed to match that data.

From Fig. 9 one can read off the combined ageSFR of any given galaxy. If one can be constrained by other information like colors, the other follows. Type is correlated with surface brightness (Table 2; de Blok et al. 1995; de Jong 1996), so it is possible to make all spirals the same old $(\sim 15 \mathrm{Gyr})$ age and satisfy the observed gas fractions simply by varying the time scale $\tau$. The variation must be quite systematic with surface brightness, and so large that one has to change the functional form of the SFR to accommodate it. (In principle, one could retain an exponential SFR by allowing $\tau \rightarrow \infty$ and then becoming negative.)

The spread of the data is large compared to the separation of the isochrones. This may indicate a large dispersion in age. More likely, it simply results from an overlap of the range of baryonic masses of any given SFR type. That is, a particular SFR type like Sd may be experienced by galaxies of a range of masses, not just one particular one as drawn in Fig. 9. Because there is inevitably overlap of this sort we do not attribute any special significance to the kink in 
the model grid for Sa and Sb SFRs at low $f_{g}$ - the overlap would wash it out, even if the models were credible at this level of detail. The lack of any corresponding feature in the data might indicate that $\tau$ does not vary as widely as assumed in the models, so that for spiral disks deviations from a constant SFR are not enormous $(\tau \gtrsim 8 \mathrm{Gyr})$.

The standard picture of galaxy evolution represented by the models of Guideroni \& Rocca-Volmerange (1987) must attribute the observed change of $f_{g}$ with surface brightness to variations in $\tau$ because $T_{G}$ is taken to be the same for all galaxies. Once this artificial boundary condition is imposed, there is no choice. It is not obvious that this is really a sensible assumption. If galaxy formation occurs from the gravitational collapse of primordial fluctuations in the density field, then the epoch of collapse of any given object depends on the density contrast of the fluctuation from which it arises. Most of the observational evidence (McGaugh 1992; 1996b) suggests that this occurs in the obvious way: large density contrasts result in high surface brightness galaxies, and small density contrasts result in low surface brightness galaxies. If this is indeed the case, one expects lower surface brightness galaxies to be younger as inferred from their colors (McGaugh \& Bothun 1994; de Blok et al. 1995). The absolute magnitude of the age difference may or may not be large; it is very difficult to put an absolute number on. From the colors, the relative shift typically appears to be on the order of a few $(3-5)$ Gyr. If high surface brightness spirals are 13 or 14 Gyr old, low surface brightness disks are roughly 8 or 10 Gyr old.

To examine this possibility in the context of the gas fraction data, consider as a trivial limiting case a scenario in which all spiral disks have the same shape star formation history. The evolutionary trajectories would all look the same in Fig. 9, and (by choice) isochrones would be horizontal lines. Instead of a systematic variation in the evolutionary time scale, real age differences would be required to explain the trend of the data. For the case of constant star formation at the same relative rate (the $\mathrm{Sd}$ track), bright galaxies with $f_{g} \approx 0.3$ would have ages $T_{G} \approx 17 \mathrm{Gyr}$, while the $\operatorname{dim}$ ones with $f_{g} \approx 0.6$ would be $T_{G} \approx 10 \mathrm{Gyr}$ old.

Either of these scenarios seem plausible, and reality is presumably more complicated - both age and evolutionary rate may play a role. Gas fractions alone do not allow us to distinguish which factor dominates.
They do show that clear differences exist. This may help lift the degeneracy that has plagued analyses of colors alone. If so, it may become possible to age-date spiral galaxies.

The distinction between a galaxy which is gas rich today because it formed early and evolved slowly or because it formed late is not clear. Some limit can be placed on these extreme cases by the plausibility of the required SFR and mean stellar age. If we insist that all galaxies have the same age, the only way to have very gas rich blue galaxies $\left(f_{g}>0.4\right)$ at $z=0$ is to have star formation rates which increase strongly with time. These retain much gas today simply because very little happened for the first half of a Hubble time. For the Im track in Fig. 9, only 10\% of the gas is consumed in the first 9 Gyr. The distinction between this and a galaxy which forms late is inobvious, and returns us to the thorny issue of the definition of galaxy formation. Perhaps a lengthy collapse by gradual dissipation and a slow evolution are intimately related.

For a more quantitative illustration, consider the conservative case of a declining star formation rate. Retaining a gas fraction greater than what is consumed in one $e$-folding $\left(f_{g}>e^{-1}=0.37\right.$, corresponding to $\mu_{0}>22$ ) requires an evolutionary time scale $\tau>T_{U}$ greater than the age of the universe, with a mean stellar age $\left\langle T_{*}\right\rangle<0.6 T_{U}$. This follows simply from requiring that the galaxy be younger than the universe: $T_{G}<T_{U}$. If we adopt a rising star formation history as suggested by the Im model in Fig. 9, the evolutionary time scale can be very (but not arbitrarily) long. This is only achieved at the expense of making the stars very young: $\left\langle T_{*}\right\rangle<1 / 4 T_{U}$, i.e., $\left\langle T_{*}\right\rangle=3$ Gyr for $T_{G}=12$ Gyr. Either way, most of the star formation in very gas rich galaxies must be weighted towards more recent epochs (cf. McGaugh \& Bothun 1994), irrespective of whether the galaxy formed early and did nothing, or formed late. Indeed, galaxies with $f_{g}>0.5$ have yet to realize most of their potential for star formation, which must be weighted towards future epochs.

\section{Conundrum}

The picture which emerges thus far seems fairly sensible, confirming and extending established wisdom about the evolution of local galaxies. Related information can be inferred from deep redshift surveys, and considerable progress has been made in measur- 
ing the properties of galaxies over an interesting range of redshift. These studies indicate a large amount of evolution in both field (Lilly et al. 1995; Ellis et al. 1996) and cluster (Schade et al. 1996) galaxies. High resolution imaging with $H S T$ shows that most of this evolution is due to morphologically late type galaxies (Glazebrook et al. 1995; Driver et al. 1995, Abraham et al. 1996). This is consistent with the evolution of the luminosity function constructed from deep surveys: red galaxies appear to evolve little, while blue ones have been fading very rapidly (Lilly et al. 1995; Ellis et al. 1996).

There is good evidence for direct evolution of the surface brightnesses of bright disks (Schade et al. $1995,1996)$. These appear to have surface brightnesses which are $\sim 1 \mathrm{mag} \operatorname{arcsec}^{-2}$ brighter at $z \approx 0.5$ than at $z=0$. This is perfectly consistent with Fig. 9: these galaxies were presumably more gas rich at $z=0.5$ and have since evolved along a track like that plotted for early-type (Sa-Sc) spirals.

The local and high redshift approaches to the evolution of early type spirals therefore give nicely consistent results. The same can not be said for late types. Many studies now indicate that the excess faint blue galaxies are predominantly late types which are evolving rapidly. If one looks locally for faint, blue, late type galaxies, one finds low surface brightness galaxies. Indeed, the similarity between the physical properties of these two populations led to the suggestion that one might account for the other (McGaugh 1994b). This similarity now extends to morphology: these galaxies literally look alike (cf. Dalcanton \& Shectman 1996). However, a simple one to one connection is not sufficient: although deep surveys are more sensitive to low surface brightness galaxies than local ones, they are still selected by flux. Magnitude limited surveys are always numerically dominated by the highest surface brightness galaxies regardless of how common low surface brightness galaxies actually are (McGaugh et al. 1995a), so for most plausible scenarios low surface brightness galaxies do not make a significant contribution to the faint counts (Ferguson \& McGaugh 1995; McLeod \& Rieke 1995) unless they evolve substantially.

It is tempting to conclude that this is exactly what happens: late type blue galaxies evolve rapidly, fading to become low surface brightness by the present epoch (Phillipps \& Driver 1995). While sensible in the context of the high redshift data, it makes no sense for the known local galaxies. Fading scenarios for the non-dwarf low surface brightness galaxies in the present sample are strongly excluded by both the colors (McGaugh \& Bothun 1994; Fig. 3) and by the gas fractions. In order to have a strongly fading trajectory (Sa or even more extreme) in Fig. 9 and retain the high observed gas fractions, such galaxies would have to be extremely young: $T_{G}<2$ Gyr for $\tau=3$ Gyr and $f_{g}>0.5$. They would not have even formed yet at the redshifts where the inferred evolution occurs.

Indeed, the evolutionary histories discussed in $\S 5$ are precisely the opposite: late type, low surface brightness galaxies evolve slowly, not rapidly. Photometrically, they evolve at roughly constant luminosity or even brighten with time. We are left with a puzzling conundrum: there exist two populations, the faint blue galaxies at high redshift and low surface brightness galaxies locally, which have very similar colors, clustering properties, and morphologies: they literally look alike, yet they can not be the same.

Perhaps the picture which emerges from the deep survey work is deceptively simple. Dividing the luminosity function into bins by color (Lilly et al. 1995) or spectral type (Heyl et al. 1996) gives the appearance of a slowly or even non-evolving red population and a rapidly evolving blue one. Yet one expects rather more evolutionary fading for red galaxies, consistent with the amount of surface brightness evolution observed by Schade et al. (1996). Perhaps there is some cross-over between color bins. This is expected at some level, as even early type galaxies should have been blue at some point early in their evolution.

It is not obvious that cross-over is adequate to explain the evolution of early types, but it may well be (Pozzetti et al. 1996). A more serious problem is understanding the rapid evolution of the blue luminosity density attributed to late types. Since the faint blue galaxies we do know about locally seem unable to explain this, perhaps the inference of a rapidly evolving dwarf population is correct and there exists locally an additional remnant population (Phillipps \& Driver 1995; Babul \& Ferguson 1996). Such a population would presumably now have faded and become red, low surface brightness, and gas poor. This is precisely the part of the $f_{g}-\mu_{0}$ plane not constrained by observation. A very large population of such objects would be required, but it is quite possible that such a population would have remained undetected so far (McGaugh 1996a), especially if the typical object is intrinsically small as indicated by deep HST imaging 
(Im et al. 1995). This picture might be considered attractive for avoiding the reverse of the gas consumption paradox: if gas rich galaxies exist as well as those near to depleting their gas supply, why are there no galaxies well past this point? The unavoidable consequence of such a scenario is that our knowledge of the local galaxy population remains glaringly incomplete.

\section{Conclusions}

Examination of the gas mass fractions of spiral galaxies over a large range in luminosity and surface brightness provides a number of insights into galaxy evolution.

1. The fraction of baryonic mass in spiral galaxies which has been converted from gas into stars is correlated with luminosity, surface brightness, and color. It is not correlated with the scale length of the disk.

2. The strongest correlation is between gas content and the central surface brightness of the disk. The surface brightness of a disk is a fundamental parameter and a good indicator of the evolutionary status of a galaxy. Dim galaxies evolve slowly, have relatively young stellar populations, and may have formed late.

3. Since luminosity traces baryonic mass and surface brightness traces surface mass density, the strength of the $f_{g}-\mu_{0}$ relation suggests that surface density is at least as important as total mass in determining the evolution of a galaxy.

4. The $f_{g}-M_{B}$ and $f_{g^{-}} \mu_{0}$ relations have the same slope. Any size evolution would cause surface brightness evolution over and above that due to luminosity evolution, leading to a difference in these slopes. The lack of such a difference indicates that spirals evolve at roughly fixed size.

5. The large number of gas rich low surface brightness galaxies resolves the gas consumption paradox.

6. The populations of local low surface brightness galaxies and high redshift faint blue galaxies are physically and morphologically similar yet grossly different in the way they evolve. This poses a difficult conundrum. One possible solution is an additional population of local, red, gas poor low surface brightness galaxies.
There is no reason to believe that we have reached the faintest limits of surface brightness, or that yet more gas rich galaxies do not exist. Given the barrier imposed by isophotal selection limits, there could certainly be more very low surface brightness galaxies that have yet to be discovered. Some of these could be even more gas rich (by extrapolation of the observed trends) and others might be gas poor $(\S 6)$.

Several further lines of work are suggested.

1. Further surveys for low surface brightness galaxies, both to deeper isophotal limits and in redder passbands. Blind $21 \mathrm{~cm}$ surveys with optical follow-up to measure surface brightnesses would also be interesting.

2. Detailed modeling of individual galaxies to match colors, metallicities, star formation rates and gas mass fractions.

3. Examining these properties on a local as well as global basis.

These projects would address some of the puzzles posed, and further advance our understanding of galaxy evolution.

In essence, we must understand how low surface brightness galaxies can remain gas rich until the present epoch. Either quiescence or youth, or some combination thereof, can be invoked. If quiescence is primarily responsible, it must be possible to suppress large scale star formation in gas disks for many Gyr, perhaps through critical density dependent phenomena (e.g., Kennicutt 1989). If youth is a factor, the existence of such unevolved galaxies suggests that the process of galaxy formation is ongoing, or has ended only recently.

We are grateful to J. M. van der Hulst and L.B. van den Hoek for helpful discussions, and thank the referee for suggesting a more expanded discussion. 
TABle 1

Data

\begin{tabular}{|c|c|c|c|c|c|c|c|c|c|c|}
\hline Galaxy & $M_{B}$ & $\mu_{0}$ & $h$ & $B-V$ & $\mathcal{T}$ & $\frac{M_{\mathrm{HI}}}{L_{B}}$ & $f_{g}^{B}$ & $\frac{M_{\mathrm{HI}}}{L_{I}}$ & $f_{g}^{I}$ & $B-I$ \\
\hline DDO 142 & -20.61 & 22.23 & 3.7 & 0.68 & 9 & 0.66 & 0.33 & $\ldots$ & $\ldots$ & $\ldots$ \\
\hline F415-3 & -16.48 & 23.87 & 1.6 & 0.52 & 9 & 1.71 & 0.64 & 1.91 & 0.68 & 1.23 \\
\hline F469-2 & -17.39 & 24.85 & 3.8 & 0.43 & 9 & 0.95 & 0.56 & 0.93 & 0.51 & 1.37 \\
\hline F530-3 & -18.77 & 23.85 & 4.9 & 0.64 & 5 & 0.53 & 0.44 & 0.46 & 0.49 & 1.49 \\
\hline F561-1 & -17.83 & 23.28 & 3.6 & 0.59 & 9 & 0.68 & 0.38 & 0.76 & 0.46 & 1.22 \\
\hline F563-1 & -17.33 & 24.00 & 4.2 & 0.65 & 9 & 2.05 & 0.62 & $\ldots$ & $\ldots$ & $\ldots$ \\
\hline F563-V1 & -16.36 & 24.29 & 2.4 & 0.56 & 10 & 0.90 & 0.47 & 1.06 & 0.55 & 1.18 \\
\hline $\mathrm{F} 563-\mathrm{V} 2$ & -18.21 & 22.22 & 2.1 & 0.36 & 10 & 0.76 & 0.55 & 0.62 & 0.42 & 1.57 \\
\hline $\mathrm{F} 565-\mathrm{V} 2$ & -15.41 & 24.73 & 2.6 & 0.53 & 10 & 2.62 & 0.73 & $\ldots$ & $\ldots$ & $\ldots$ \\
\hline $\mathrm{F} 567-2$ & -17.38 & 24.41 & 5.7 & 0.67 & 9 & 1.56 & 0.54 & 2.32 & 0.72 & 0.92 \\
\hline F568-1 & -18.12 & 23.76 & 5.3 & 0.62 & 5 & 1.45 & 0.69 & 1.49 & 0.75 & 1.32 \\
\hline F568-3 & -18.31 & 23.07 & 4.0 & 0.55 & 7 & 0.85 & 0.51 & 0.90 & 0.55 & 1.29 \\
\hline F568-6 & -21.57 & 23.60 & 21.1 & 0.63 & 5 & 0.44 & 0.32 & 0.19 & 0.28 & 2.26 \\
\hline F568-V1 & -17.88 & 23.29 & 3.2 & 0.51 & 8 & 1.11 & 0.56 & 1.26 & 0.60 & 1.21 \\
\hline F571-5 & -17.14 & 23.65 & 2.9 & 0.34 & 9 & 1.55 & 0.73 & $\ldots$ & $\ldots$ & $\ldots$ \\
\hline F571-V1 & -17.04 & 23.99 & 3.2 & 0.53 & 8 & 1.13 & 0.56 & $\ldots$ & $\ldots$ & $\ldots$ \\
\hline F574-2 & -17.64 & 24.33 & 6.0 & 0.63 & 9 & 0.92 & 0.44 & $\ldots$ & $\ldots$ & $\ldots$ \\
\hline F577-V1 & -18.21 & 23.94 & 5.7 & 0.38 & 7 & 0.83 & 0.61 & $\ldots$ & $\ldots$ & $\ldots$ \\
\hline F611-1 & -15.76 & 24.65 & 2.0 & 0.44 & 10 & 1.07 & 0.58 & 0.99 & 0.53 & 1.44 \\
\hline F615-1 & -17.54 & 23.36 & 2.8 & 0.51 & 10 & 0.61 & 0.40 & 0.58 & 0.40 & 1.40 \\
\hline F746-1 & -19.42 & 23.24 & 4.0 & 0.65 & 10 & 0.73 & 0.37 & 0.71 & 0.45 & 1.38 \\
\hline Malin 1 & -22.30 & 26.60 & 73.0 & 0.90 & $\ldots$ & 3 & 0.75 & $\ldots$ & $\ldots$ & $\ldots$ \\
\hline NGC 3913 & -19.05 & 22.60 & 2.1 & 0.60 & 7 & 0.29 & 0.24 & $\ldots$ & $\ldots$ & $\ldots$ \\
\hline NGC 4411A & -19.13 & 22.07 & 1.7 & 0.68 & 8 & 0.34 & 0.21 & $\ldots$ & $\ldots$ & $\ldots$ \\
\hline NGC 4411B & -19.65 & 22.07 & 2.2 & 0.56 & 8 & 0.33 & 0.25 & $\ldots$ & $\ldots$ & $\ldots$ \\
\hline NGC 5774 & -20.55 & 22.35 & 3.8 & 0.54 & 7 & 0.86 & 0.51 & $\ldots$ & $\ldots$ & $\ldots$ \\
\hline UGC 89 & -21.50 & 22.07 & 8.7 & 0.74 & 1 & 0.13 & 0.26 & 0.09 & 0.29 & 1.67 \\
\hline UGC 93 & -20.06 & 22.33 & 7.0 & 0.64 & 8 & 0.78 & 0.41 & 0.54 & 0.38 & 1.75 \\
\hline UGC 128 & -18.78 & 24.22 & 6.8 & 0.51 & 6 & 1.24 & 0.66 & 1.57 & 0.72 & 1.09 \\
\hline UGC 334 & -18.99 & 23.36 & 6.6 & 0.90 & 9 & 0.92 & 0.32 & 1.02 & 0.51 & 1.24 \\
\hline UGC 438 & -21.10 & 20.45 & 4.2 & 0.73 & 5 & 0.15 & 0.16 & 0.09 & 0.15 & 1.89 \\
\hline UGC 463 & -20.74 & 20.76 & 4.0 & $\ldots$ & 5 & 0.17 & $\ldots$ & 0.11 & 0.18 & 1.72 \\
\hline UGC 490 & -20.57 & 21.47 & 5.6 & 0.86 & 5 & 0.63 & 0.38 & 0.34 & 0.41 & 2.00 \\
\hline UGC 508 & -21.56 & 22.05 & 9.5 & 0.92 & 2 & 0.17 & 0.22 & 0.09 & 0.24 & 2.01 \\
\hline UGC 1230 & -18.33 & 23.36 & 5.3 & 0.52 & 5 & 1.72 & 0.77 & 1.98 & 0.80 & 1.20 \\
\hline UGC 1305 & -20.30 & 22.02 & 6.0 & 0.93 & 4 & 0.16 & 0.15 & 0.07 & 0.14 & 2.24 \\
\hline UGC 1551 & -19.46 & 22.47 & 4.6 & 0.62 & 8 & 0.49 & 0.31 & 0.43 & 0.31 & 1.48 \\
\hline UGC 1577 & -20.64 & 22.44 & 7.7 & 0.93 & 4 & 0.38 & 0.30 & 0.22 & 0.34 & 1.95 \\
\hline UGC 1719 & -21.35 & 22.45 & 11.6 & 0.82 & 3 & 0.27 & 0.31 & 0.16 & 0.31 & 1.90 \\
\hline UGC 1792 & -20.36 & 21.65 & 5.6 & 0.85 & 5 & 0.61 & 0.38 & 0.46 & 0.44 & 1.65 \\
\hline UGC 2064 & -19.97 & 22.28 & 5.6 & 0.88 & 4 & 0.47 & 0.36 & 0.25 & 0.35 & 2.01 \\
\hline UGC 2124 & -19.32 & 22.34 & 3.9 & 0.98 & 1 & 0.63 & 0.56 & 0.22 & 0.51 & 2.50 \\
\hline UGC 2125 & -20.37 & 23.20 & 9.1 & 0.85 & 5 & 0.54 & 0.35 & 0.37 & 0.39 & 1.74 \\
\hline UGC 2197 & -19.95 & 22.57 & 6.1 & 0.73 & 6 & 0.64 & 0.39 & 0.48 & 0.38 & 1.66 \\
\hline UGC 2368 & -19.89 & 23.67 & 6.6 & 0.70 & 3 & 0.70 & 0.58 & 0.48 & 0.55 & 1.76 \\
\hline
\end{tabular}


TABLE 1-Continued

\begin{tabular}{|c|c|c|c|c|c|c|c|c|c|c|}
\hline Galaxy & $M_{B}$ & $\mu_{0}$ & $h$ & $B-V$ & $\mathcal{T}$ & $\frac{M_{\mathrm{HI}}}{L_{B}}$ & $f_{g}^{B}$ & $\frac{M_{\mathrm{HI}}}{L_{I}}$ & $f_{g}^{I}$ & $B-I$ \\
\hline UGC 3066 & -19.96 & 22.03 & 4.3 & 0.73 & 7 & 0.73 & 0.38 & 0.44 & 0.31 & 1.88 \\
\hline UGC 3080 & -19.55 & 21.99 & 3.8 & 0.65 & 5 & 0.67 & 0.49 & 0.47 & 0.46 & 1.72 \\
\hline UGC 4126 & -20.89 & 21.83 & 6.9 & 0.63 & 3 & 0.21 & 0.32 & 0.24 & 0.30 & 1.62 \\
\hline UGC 4256 & -21.09 & 21.18 & 5.9 & 0.49 & 5 & 0.32 & 0.40 & 0.13 & 0.28 & 1.82 \\
\hline UGC 4308 & -20.29 & 21.34 & 4.5 & 0.59 & 5 & 0.35 & 0.37 & 0.26 & 0.33 & 1.68 \\
\hline UGC 4368 & -20.01 & 21.52 & 4.3 & 0.57 & 6 & 0.55 & 0.44 & 0.38 & 0.38 & 1.76 \\
\hline UGC 4422 & -21.02 & 22.04 & 8.5 & 0.47 & 5 & 0.26 & 0.38 & 0.17 & 0.27 & 1.77 \\
\hline UGC 4458 & -21.02 & 21.72 & 5.9 & 0.84 & 1 & 0.21 & 0.32 & 0.12 & 0.34 & 1.96 \\
\hline UGC 4841 & -19.84 & 22.80 & 3.3 & 0.67 & 7 & 0.54 & 0.34 & $\ldots$ & $\ldots$ & $\ldots$ \\
\hline UGC 4922 & -19.88 & 23.13 & 4.0 & 0.57 & 7 & 0.60 & 0.41 & $\ldots$ & $\ldots$ & $\ldots$ \\
\hline UGC 5303 & -19.05 & 21.32 & 3.1 & 0.65 & 5 & 0.24 & 0.26 & 0.17 & 0.26 & 1.69 \\
\hline UGC 5510 & -18.77 & 20.66 & 1.6 & $\ldots$ & 4 & 0.38 & $\ldots$ & 0.37 & 0.47 & 1.38 \\
\hline UGC 5554 & -18.15 & 20.98 & 1.4 & $\ldots$ & 1 & 0.12 & $\ldots$ & 0.08 & 0.26 & 1.82 \\
\hline UGC 5633 & -17.04 & 23.13 & 2.3 & 0.61 & 8 & 1.03 & 0.49 & $\ldots$ & $\ldots$ & $\ldots$ \\
\hline UGC 5675 & -16.31 & 23.77 & 2.0 & $\ldots$ & 9 & $\ldots$ & $\ldots$ & 0.79 & 0.47 & 1.80 \\
\hline UGC 5709 & -19.77 & 23.60 & 5.4 & 0.48 & 5 & 0.52 & 0.52 & 0.33 & 0.41 & 1.83 \\
\hline UGC 5842 & -18.54 & 21.44 & 2.3 & $\ldots$ & 6 & $\ldots$ & $\ldots$ & 0.21 & 0.25 & 1.49 \\
\hline UGC 6151 & -17.49 & 23.26 & 2.8 & 0.51 & 9 & 0.64 & 0.41 & 0.61 & 0.41 & 1.41 \\
\hline UGC 6277 & -19.08 & 20.88 & 2.0 & $\ldots$ & 5 & 0.25 & $\ldots$ & 0.18 & 0.27 & 1.69 \\
\hline UGC 6445 & -18.39 & 20.55 & 1.2 & 0.64 & 4 & 0.59 & 0.51 & 0.35 & 0.47 & 1.91 \\
\hline UGC 6453 & -18.38 & 20.91 & 1.5 & 0.89 & 4 & 0.58 & 0.40 & 0.47 & 0.54 & 1.56 \\
\hline UGC 6614 & -20.29 & 24.85 & 15.9 & 0.72 & 5 & 0.97 & 0.55 & 0.39 & 0.45 & 2.32 \\
\hline UGC 6693 & -21.33 & 21.60 & 8.3 & 0.69 & 4 & 0.43 & 0.42 & 0.29 & 0.43 & 1.76 \\
\hline UGC 6746 & -21.46 & 21.38 & 7.8 & 0.81 & 1 & 0.14 & 0.29 & $\ldots$ & $\ldots$ & $\ldots$ \\
\hline UGC 6922 & -17.76 & 23.52 & 1.8 & 0.61 & 10 & 0.51 & 0.31 & $\cdots$ & $\cdots$ & $\cdots$ \\
\hline UGC 6956 & -17.69 & 23.70 & 1.9 & 0.56 & 9 & 0.52 & 0.33 & $\ldots$ & $\ldots$ & $\ldots$ \\
\hline UGC 6983 & -19.43 & 22.77 & 2.7 & 0.54 & 6 & 0.78 & 0.54 & $\ldots$ & $\ldots$ & $\ldots$ \\
\hline UGC 7169 & -19.67 & 20.11 & 1.8 & 0.40 & 5 & 0.36 & 0.48 & $\ldots$ & $\ldots$ & $\ldots$ \\
\hline UGC 7315 & -17.67 & 19.99 & 0.8 & 0.69 & 4 & 0.06 & 0.09 & 0.03 & 0.08 & 2.02 \\
\hline UGC 7450 & -21.43 & 21.17 & 6.4 & 0.59 & 4 & 0.10 & 0.17 & 0.08 & 0.17 & 1.58 \\
\hline UGC 7523 & -18.58 & 21.49 & 2.0 & 0.70 & 3 & 0.05 & 0.09 & 0.03 & 0.09 & 1.93 \\
\hline UGC 7557 & -19.12 & 22.88 & 2.5 & 0.41 & 10 & 0.78 & 0.52 & $\ldots$ & $\ldots$ & $\ldots$ \\
\hline UGC 7685 & -19.42 & 22.89 & 2.9 & 0.65 & 10 & 0.72 & 0.37 & $\ldots$ & $\ldots$ & $\ldots$ \\
\hline UGC 7901 & -18.62 & 20.07 & 1.2 & 0.63 & 5 & 0.34 & 0.34 & 0.23 & 0.31 & 1.77 \\
\hline UGC 7911 & -19.36 & 22.92 & 2.8 & 0.48 & 9 & 0.42 & 0.33 & $\ldots$ & $\ldots$ & $\ldots$ \\
\hline UGC 8279 & -19.66 & 20.52 & 2.3 & $\ldots$ & 5 & 0.27 & $\ldots$ & 0.19 & 0.29 & 1.68 \\
\hline UGC 8289 & -20.91 & 21.80 & 7.1 & $\ldots$ & 4 & 0.32 & $\ldots$ & 0.27 & 0.41 & 1.50 \\
\hline UGC 8865 & -19.93 & 21.89 & 5.0 & & 2 & 0.22 & & 0.15 & 0.37 & 1.72 \\
\hline UGC 9024 & -18.73 & 24.66 & 7.5 & $\ldots$ & 9 & 0.82 & $\ldots$ & 0.89 & 0.50 & 1.25 \\
\hline UGC 9061 & -22.27 & 22.63 & 21.9 & $\ldots$ & 4 & 0.33 & $\cdots$ & 0.19 & 0.32 & 1.95 \\
\hline UGC 9481 & -20.22 & 21.22 & 4.3 & $\ldots$ & 4 & 0.52 & $\ldots$ & 0.42 & 0.52 & 1.57 \\
\hline UGC 9926 & -20.09 & 20.13 & 2.3 & 0.63 & 5 & 0.19 & 0.22 & $\ldots$ & $\ldots$ & $\ldots$ \\
\hline UGC 9943 & -19.92 & 20.40 & 2.6 & 0.67 & 5 & 0.21 & 0.23 & 0.14 & 0.22 & 1.80 \\
\hline UGC 10083 & -19.79 & 21.51 & 2.9 & 0.42 & 2 & 0.27 & 0.55 & 0.27 & 0.49 & 1.34 \\
\hline UGC 10437 & -18.45 & 24.03 & 5.9 & 0.29 & 7 & 1.10 & 0.73 & 1.05 & 0.59 & 1.40 \\
\hline
\end{tabular}


TABLE 1-Continued

\begin{tabular}{cccccrccccc}
\hline \hline Galaxy & \multicolumn{1}{c}{$M_{B}$} & \multicolumn{1}{c}{$\mu_{0}$} & \multicolumn{1}{c}{$h$} & $B-V$ & $\mathcal{T}$ & $\frac{M_{\mathrm{HI}}}{L_{B}}$ & $f_{g}^{B}$ & $\frac{M_{\mathrm{HI}}}{L_{I}}$ & $f_{g}^{I}$ & $B-I$ \\
\hline UGC 10584 & -21.20 & 21.77 & 8.3 & 0.61 & 5 & 0.55 & 0.47 & 0.38 & 0.44 & 1.76 \\
UGC 11628 & -21.36 & 22.27 & 11.0 & 0.96 & 2 & 0.26 & 0.29 & 0.18 & 0.36 & 1.76 \\
UGC 11708 & -20.65 & 21.51 & 5.0 & 0.66 & 5 & 0.27 & 0.25 & 0.16 & 0.21 & 1.87 \\
UGC 11868 & -18.56 & 22.65 & 1.7 & 0.74 & 10 & 0.42 & 0.22 & $\ldots$ & $\ldots$ & $\ldots$ \\
UGC 11872 & -19.46 & 20.46 & 1.5 & 0.84 & 3 & 0.21 & 0.24 & 0.11 & 0.23 & 2.03 \\
UGC 12151 & -17.76 & 23.27 & 3.1 & 0.55 & 10 & 1.45 & 0.59 & 1.47 & 0.61 & 1.33 \\
UGC 12343 & -20.93 & 21.95 & 8.3 & 0.98 & 5 & 0.26 & 0.18 & 0.14 & 0.22 & 1.98 \\
UGC 12379 & -21.37 & 21.99 & 8.0 & 0.80 & 4 & 0.42 & 0.36 & 0.20 & 0.31 & 2.14 \\
UGC 12391 & -20.47 & 21.50 & 5.1 & 0.64 & 5 & 0.73 & 0.52 & 0.48 & 0.48 & 1.79 \\
UGC 12511 & -19.76 & 22.46 & 5.8 & 0.72 & 6 & 1.52 & 0.61 & 0.76 & 0.55 & 2.09 \\
UGC 12614 & -20.91 & 20.94 & 5.1 & 0.73 & 5 & 0.20 & 0.20 & 0.14 & 0.22 & 1.71 \\
UGC 12638 & -20.72 & 22.17 & 9.1 & 0.75 & 5 & 0.30 & 0.25 & 0.21 & 0.26 & 1.76 \\
UGC 12654 & -20.17 & 21.76 & 5.3 & 0.78 & 4 & 0.44 & 0.38 & 0.31 & 0.43 & 1.72 \\
UGC 12695 & -18.92 & 24.59 & 8.4 & 0.37 & 9 & 1.28 & 0.67 & 1.31 & 0.60 & 1.32 \\
UGC 12754 & -18.74 & 21.81 & 3.1 & 0.54 & 6 & 0.37 & 0.35 & 0.36 & 0.36 & 1.37 \\
UGC 12776 & -21.25 & 23.39 & 20.3 & $\ldots$ & 3 & 0.38 & $\ldots$ & 0.29 & 0.45 & 1.62 \\
UGC 12808 & -21.41 & 20.31 & 3.9 & 0.70 & 3 & 0.09 & 0.16 & 0.05 & 0.15 & 1.85 \\
UGC 12845 & -20.19 & 22.69 & 8.0 & 0.89 & 7 & 0.79 & 0.33 & 0.56 & 0.42 & 1.72 \\
\hline
\end{tabular}

TABLE 2

Correlation Matrix

\begin{tabular}{lcccrrr}
\hline \hline & $f_{g}$ & $M_{B}$ & $\mu_{0}$ & $\log (h)$ & $B-V$ & \multicolumn{1}{c}{} \\
\hline $\log \left(\frac{M_{\mathrm{HI}}}{L_{B}}\right)$ & \multirow{2}{*}{0.86} & 0.58 & 0.73 & 0.00 & -0.37 & 0.64 \\
$f_{g}$ & & 0.51 & 0.63 & 0.03 & -0.55 & 0.36 \\
$M_{B}$ & & & 0.58 & -0.59 & -0.49 & 0.68 \\
$\mu_{0}$ & & & & 0.18 & -0.35 & 0.59 \\
$\log (h)$ & & & & & 0.33 & -0.37 \\
$B-V$ & & & & & & -0.45 \\
\hline
\end{tabular}


TABLE 3

Star Formation Histories

\begin{tabular}{|c|c|c|c|}
\hline & & Exponential $^{\mathrm{a}}$ & Power Law ${ }^{b}$ \\
\hline Star Formation Rate ${ }^{\mathrm{c}, \mathrm{d}}$ & $\dot{f}_{g}=$ & $-\tau^{-1} e^{-t / \tau}$ & $-\frac{x+1}{\tau_{g}}\left(\frac{t}{\tau_{g}}\right)^{x}$ \\
\hline Gas Mass Fraction & $f_{g}=$ & $e^{-t / \tau}$ & $1-\left(\frac{t}{\tau_{g}}\right)^{x+1}$ \\
\hline Galaxy Age $\mathrm{e}^{\mathrm{e}}$ & $T_{G}=$ & $-\tau \ln f_{g}$ & $\tau_{g}\left(1-f_{g}\right)^{1 /(x+1)}$ \\
\hline Mean Stellar Age & $\left\langle T_{*}\right\rangle=$ & $-\tau\left(1+\frac{\ln f_{g}}{1-f_{g}}\right)$ & $T_{G} /(x+2)$ \\
\hline Time Scale Limit ${ }^{c, f}$ & & $\tau>-T_{U} / \ln f_{g}$ & $\tau_{g}<T_{U}\left(1-f_{g}\right)^{-1 /(x+1)}$ \\
\hline Mean Stellar Age Limit & $\left\langle T_{*}\right\rangle<$ & $T_{U}\left(\frac{1}{\ln f_{g}}+\frac{1}{1-f_{g}}\right)$ & $T_{U} /(x+2)$ \\
\hline
\end{tabular}

a Declining SFR with $\tau>0$.

${ }^{\mathrm{b}}$ Flat or rising SFR with $\tau_{g}$ and $x \geq 0$.

${ }^{\mathrm{c}} \tau=e$-folding time scale; $\tau_{g}=$ gas consumption time scale.

${ }^{\mathrm{d}}$ Global SFR normalized by total baryonic mass.

${ }^{\mathrm{e}} T_{G}$ defined by the onset of star formation $=$ age of oldest stars.

${ }^{\mathrm{f}}$ Limits obtained by requiring $T_{G}<T_{U}$, the age of the universe.

TABLE 4

Population Models

\begin{tabular}{cccl}
\hline \hline $\mathcal{T}$ & $\tau($ Gyr $)$ & $x$ & \multicolumn{1}{c}{ Form of SFR } \\
\hline $\mathrm{Sa}$ & 3.3 & $\cdots$ & Exponential decay \\
$\mathrm{Sb}$ & 5 & $\cdots$ & Exponetial decay \\
$\mathrm{Sc}$ & 10 & $\cdots$ & Exponetial decay \\
$\mathrm{Sd}$ & 20 & 0 & Constant \\
$\mathrm{Im}$ & 20 & 2 & Rising power law \\
\hline
\end{tabular}

Note.-Hubble type is used here to denote the models of Guideroni \& RoccaVolmerange (1987). Strictly speaking, these only specify the shape of the model star formation history. 


\section{REFERENCES}

Abraham, R. G., Tanvir, N. R., Santiago, B. X., Ellis, R. S., Glazebrook, K., \& van den Bergh, S. 1996, MNRAS, 279, 47

Babul, A., \& Ferguson, H. C. 1996, ApJ, 458, 100

Bergvall, N., \& Rönnback, J. 1995, MNRAS, 273, 603

Bruzual, A. G., \& Charlot, S. 1993, ApJ, 405, 538

Bothun, G. D. 1985, AJ, 90, 1982

Bothun, G. D., Impey, C. D., Malin, D. F., \& Mould, J. R. 1987, AJ, 94, 23

Bothun, G. D., Schombert, J. M., Impey, C. D., Sprayberry, D., \& McGaugh, S. S. 1993, AJ, 106, 530

Bottema, R. 1993, A\&A, 275, 16

Bottema, R. 1995, Ph.D. thesis, University of Groningen

Dalcanton, J. J. 1993, ApJ, 415, L87

Dalcanton, J. J., \& Shectman, S. A. 1996, ApJ, 465, L9

de Blok, W. J. G., van der Hulst, J. M., \& Bothun, G. D. 1995, MNRAS, 274, 235

de Blok, W. J. G., \& McGaugh, S. S. 1996, ApJ, 469, L89

de Blok, W. J. G., \& McGaugh, S. S. 1997, MNRAS, submitted

de Blok, W. J. G., McGaugh, S. S., \& van der Hulst, J. M. 1996, MNRAS, in press

de Jong, R. S. 1995, Ph.D. thesis, University of Groningen

de Jong, R. S. 1996, A\&A, 313, 45

de Jong, R. S., \& van der Kruit, P. C. 1994, A\&AS, 106,451

Disney, M. J., \& Phillipps, S. 1983, MNRAS, 205, 1253

Driver, S., Windhorst, R. A., \& Griffiths, R. E. 1995 453,48

Ellis, R. S., Colless, M., Broadhurst, T. J., Heyl, J. S., \& Glazebrook, K. 1996, MNRAS, 280, 235

Ferguson, H. C., \& McGaugh, S. S. 1995, ApJ, 440, 470

Im, M., Ratnatunga, K. U., Griffiths, R. E., \& Casertano, S. 1995, ApJ, 445, L15
Impey, S. D., \& Bothun, C. D. 1989, ApJ, 341, 89

Impey, S. D., Sprayberry, D., Irwin, M., \& Bothun, C. D. 1996, preprint

Guideroni \& Rocca-Volmerange 1987, A\&A, 186, 1

Glazebrook, K., Ellis, R., Santiago, B., \& Griffiths, R. 1995, MNRAS, 275, L19

Heyl, J., Colless, M., Ellis, R. S., \& Broadhurst, T. 1996, MNRAS, in press

Kennicutt, R. C. 1989, ApJ, 344, 685

Kennicutt, R. C., Tamblyn, P., \& Congdon, C. E. 1994, ApJ, 435, 22

Lilly, S. J., Tresse, L., Hammer, F., Crampton, D., \& Le Fèvre, O. 1995, ApJ, 455, 108

McGaugh, S. S. 1992, Ph.D. thesis, University of Michigan

McGaugh, S. S. 1994a, ApJ, 426, 135

McGaugh, S. S. 1994b, Nature, 367, 538

McGaugh, S. S. 1996a, MNRAS, 280, 337

McGaugh, S. S. 1996b, in IAU Symposium 171: New Light on Galaxy Evolution, eds. R. Bender \& R. L. Davies, (Dordrecht: Kluwer), 97

McGaugh, S. S., \& Bothun, G. D. 1994, AJ, 107, 530

McGaugh, S. S., Bothun, G. D., \& Schombert, J. M. 1995a, AJ, 110, 573

McGaugh, S. S., Schombert, J. M., \& Bothun, G. D. 1995b, AJ, 109, 2019

McLeod, B. A., \& Rieke, M. J. 1995, ApJ, 454, 611

Mo, H. J., McGaugh, S. S., \& Bothun, G. D. 1994, MNRAS, 267, 129

Naim, A., Lahav, O., Sodré, L., Storrie-Lombardi, M. C. 1995, MNRAS, 275, 567

Phillipps, S., \& Driver, S. 1995, MNRAS, 274, 832

Pozzetti, L., Bruzual, G., \& Zamorani, G. 1996, MNRAS, in press

Rao, S. \& Briggs, F. 1993, ApJ, 419, 515

Roberts, M. S. 1963, ARA\&A, 1, 149

Romanishin, W., Krumm, N., Salpeter, E. E., Knapp, G. R., Strom, K. M., \& Strom, S. E. 1982, ApJ, 263, 94

Romanishin, W., Strom, K. M., \& Strom, S. E. 1983, ApJS, 53, 105

Rönnback, J., \& Bergvall, N. 1994, A\&AS, 108, 193 
Rönnback, J., \& Bergvall, N. 1995, A\&A, 302, 353

Salpeter, E. E., \& Hoffman, G. L. 1996, 465, 595

Schade, D., Carlberg, R. G., Yee, H. K. C., Lopez-

Cruz, O., \& Ellingson, E. 1996, ApJ, 465, 103

Schade, D., Lilly, S. J., Crampton, D., Hammer, F., Le Fèvre, O., \& Tresse, L. 1995, ApJ, 451, L1

Schombert, J. M., Bothun, G. D., Impey, C. D., \& Mundy, L. G. 1990, AJ, 100, 1523

Schombert, J. M., Bothun, G. D., Schneider, S. E., \& McGaugh, S. S. 1992, AJ, 103, 1107

Tinsley, B. M. 1968, ApJ, 151, 547

van der Hulst, J. M., Skillman, E. D., Smith, T. R., Bothun, G. D., McGaugh, S. S., \& de Blok, W. J. G. 1993, AJ, 106, 548

Wilson, C. D. 1995, ApJ, 448, L97

Worthey, G. 1994, ApJS, 95, 107

Young, J. S., \& Knezek, P. M. 1989, ApJ, 347, L55

This 2-column preprint was prepared with the AAS LATEX macros v4.0.
Fig. 1.- The HI mass to light ratio as a function of disk central surface brightness. a) $B$-band b) $I$ band. There is a strong correlation between the gas content of spiral galaxies and their surface brightness. This is apparent in both passbands even though there are fewer data in $I$. What scatter there is reflects real differences between galaxies as the uncertainties are small compared to the range plotted: typically 0.2 - 0.3 mag. in $\mu_{0}$, and 0.2 dex in the distance independent flux ratio $M_{\mathrm{HI}} / L_{B}$. Note the sharp end to the data as $\mu_{0} \rightarrow 25 \mathrm{~B}$ mag $\operatorname{arcsec}^{-2}$ imposed by isophotal selection limits. Without our data for low surface brightness galaxies, this occurs brighter than $\mu_{0} \approx 23$ in (a). This is also apparent in the gap in (b) at $\mu_{0}{ }^{I} \approx 21$. Examining only galaxies brighter than this conceals the correlation for lack of dynamic range. The very low surface brightness giant Malin 1 [the triangle in (a)] breaks this isophotal barrier because it was serendipitously discovered, in part due to its high gas content.

Fig. 2.- The optical ( $B$-band) properties of spiral galaxies: a) central surface brightness vs. absolute magnitude and b) the scale length length vs. absolute magnitude. Different symbols represent different Hubble types, progressing from early to late types in order of increasing number of points on the symbol [key inset in (a)].

Fig. 3.- I-band central surface brightness $\mu_{0}{ }^{I}=$ $\mu_{0}-(B-I)$ vs. $V-I$ color. Symbols correspond to Hubble type as per Fig. 2. Note that lower surface brightness disks are systematically bluer than those of higher surface brightness - they are not the result of fading after cessation of star formation.

Fig. 4.- The vertical velocity dispersion of spiral disks as a function of color. Data are from Bottema $(1993 ; 1995)$. The line is our estimator for $\Upsilon_{*}$ mapped onto the velocity dispersion by equation (8) of Bottema (1993). The normalization is chosen to match the data, but the shape of the line is not a fit thereto (see de Blok \& McGaugh 1997). Nevertheless, it does match the observed trend, which goes in the expected sense that redder galaxies have higher $\Upsilon_{*}$.

Fig. 5.- The stellar mass of sample galaxies (in solar masses) computed from $\mathcal{M}_{*}=\Upsilon_{*} L$ independently in the $B$ (abscissa) and $I$ (ordinate) bands. Separate measures of the luminosity $\left(L_{B}\right.$ and $\left.L_{I}\right)$ are used together with the estimators for $\Upsilon_{*}$ discussed in the 
text. The data closely follow the line of unity, indicating reasonable agreement between the two methods.

Fig. 6. - The ratio of molecular to atomic gas mass as a function of Hubble type. Data (mean and error in the mean) are from Young \& Knezek (1989). As usual, the error in the mean understates the amount of real scatter at a given type. The line is our fit to the data (see text).

Fig. 7.- The gas fraction of spiral galaxies plotted against a) absolute $B$ magnitude, b) disk central surface brightness, c) disk scale length and d) color. The full possible range of $0 \leq f_{g} \leq 1$ is plotted and for the first time is needed - the dimmest galaxies are mostly gaseous with $f_{g} \gtrsim 0.5$. A strong relation exists between $f_{g}$ and $M_{B}, \mu_{0}$, and color. There is no relation at all between $f_{g}$ and the size of the disk. Note that the $f_{g}-M_{B}$ and $-\mu_{0}$ relations have the same slope. Malin 1 (triangle) is an extreme case with a gas fraction which is abnormally high for its luminosity but normal for its surface brightness.

Fig. 8. - Like Fig. 7 except for the $I$-band data. The figure shows $f_{g}^{I}$ against a) absolute $I$ magnitude, b) $I$-band disk central surface brightness, c) scale length, and d) $V-I$ color. The gas fraction is determined independently from that in Fig. 7 by assuming that $\Upsilon_{*}^{I}$ is constant for all spirals. This gives indistinguishable results from the $B$-band even though a completely different estimator for the stellar mass has been employed.

Fig. 9.- The data from Fig. 7 (a) and (b) overplotted after shifting by the intercepts of equation (3). Circles: $M_{B}$. Squares: $\mu_{0}$. Lines are model evolutionary tracks from Guideroni \& Rocca-Volmerange (1987). Horizontal lines are isochrones every 2 Gyr from 1 to 15 Gyr. Vertical lines are evolutionary trajectories for specific star formation histories, labeled by the Hubble type the model attempts to represent. Early types (Sa to Sc) have exponentially declining star formation rates, while Sd is constant and Im rising with time (Table 4). The position of these lines depends on the total baryonic mass of a galaxy. They are drawn with separations corresponding to the mean of each type as stipulated by Guideroni \& Rocca-Volmerange (1987); the absolute position of the resulting grid has been adjusted to coincide with the data. Though these (or any) population synthesis models are by no means perfect, they do illustrate how $f_{g}$ constrains the combination of evolutionary rate and age. With a large dynamic range in $f_{g}$ and the traditional constraints of color and metallicity, it may be possible to construct a multidimensional extragalactic analog to the HR diagram which might enable the age-dating of spiral galaxies. 


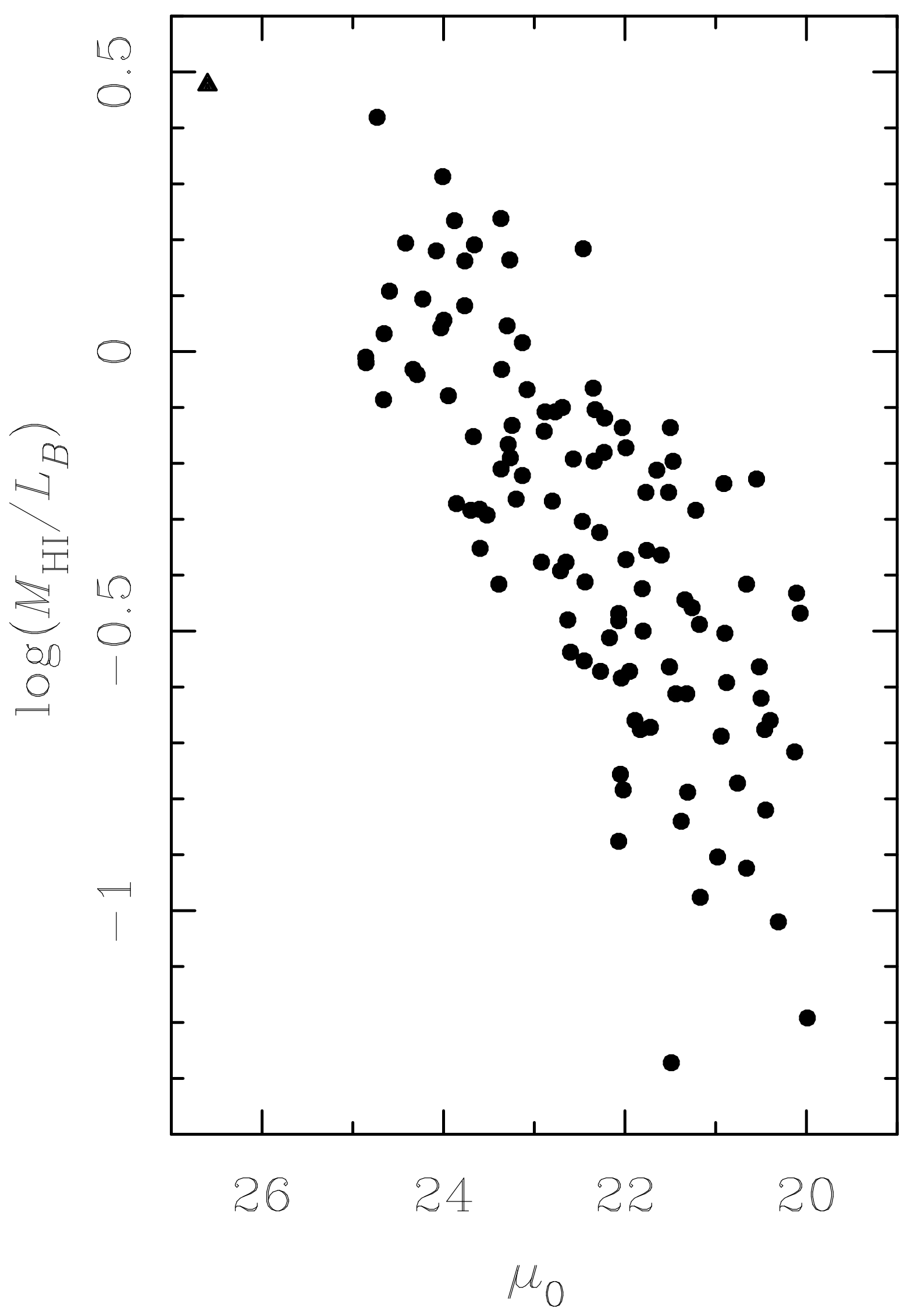




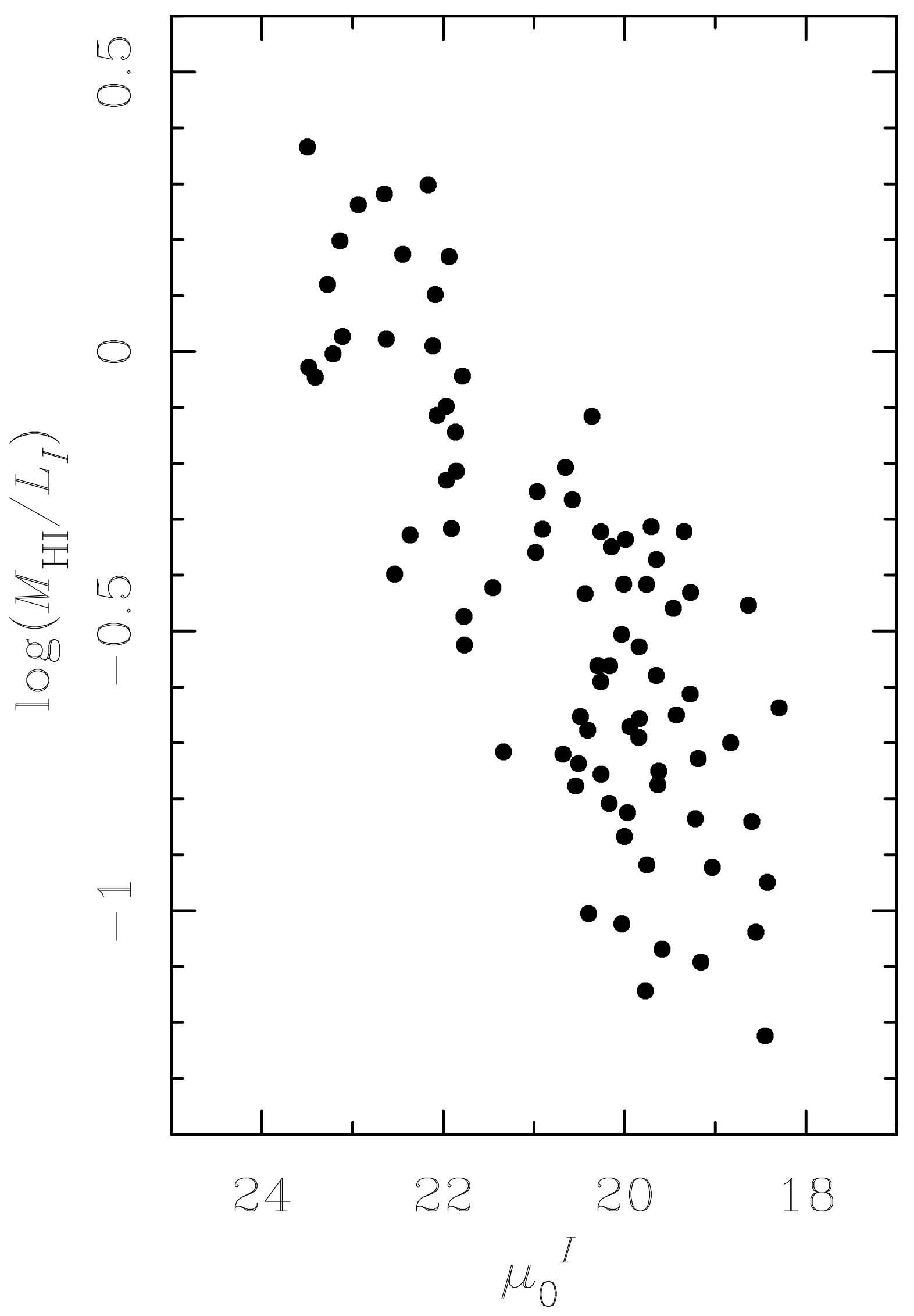




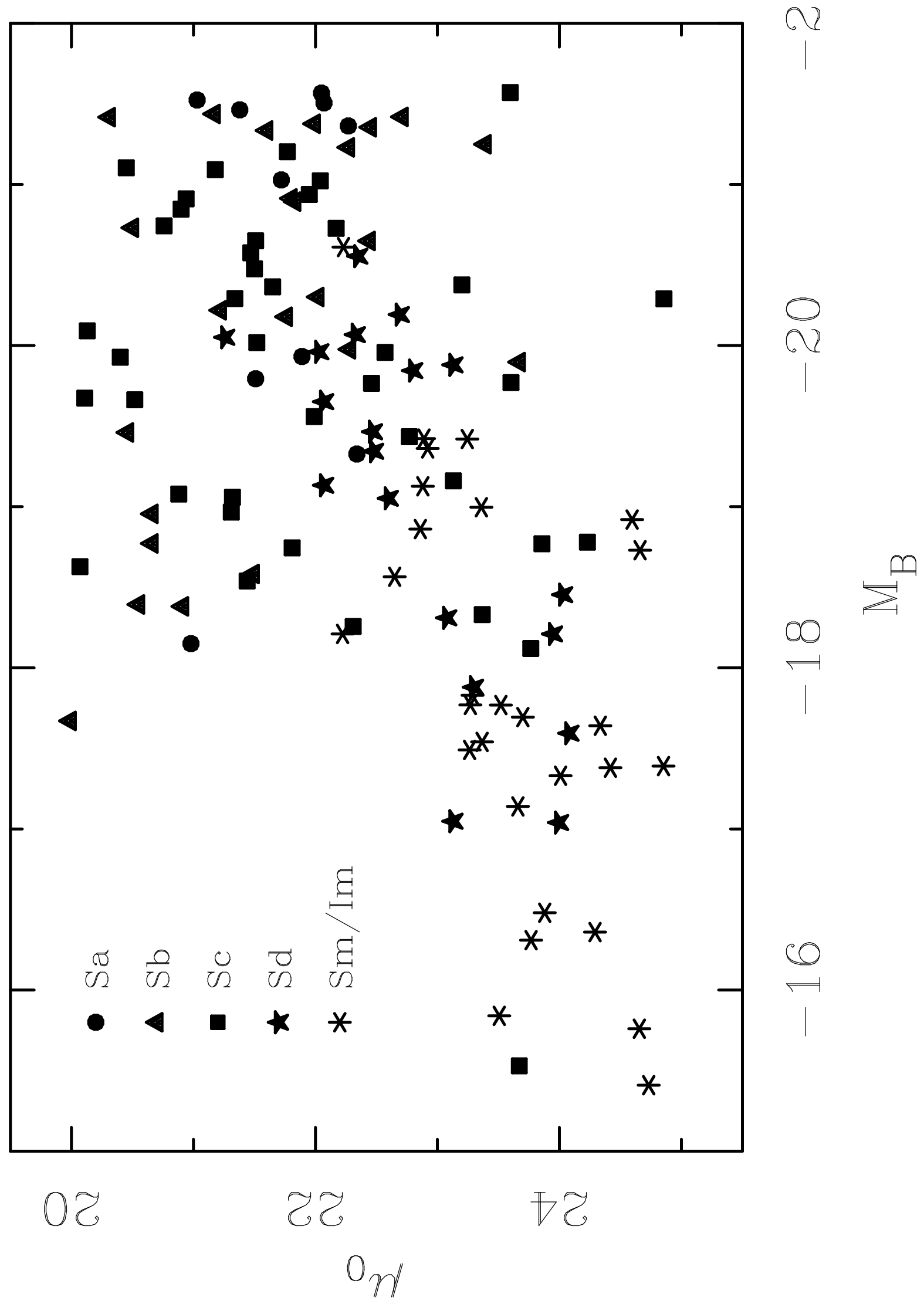




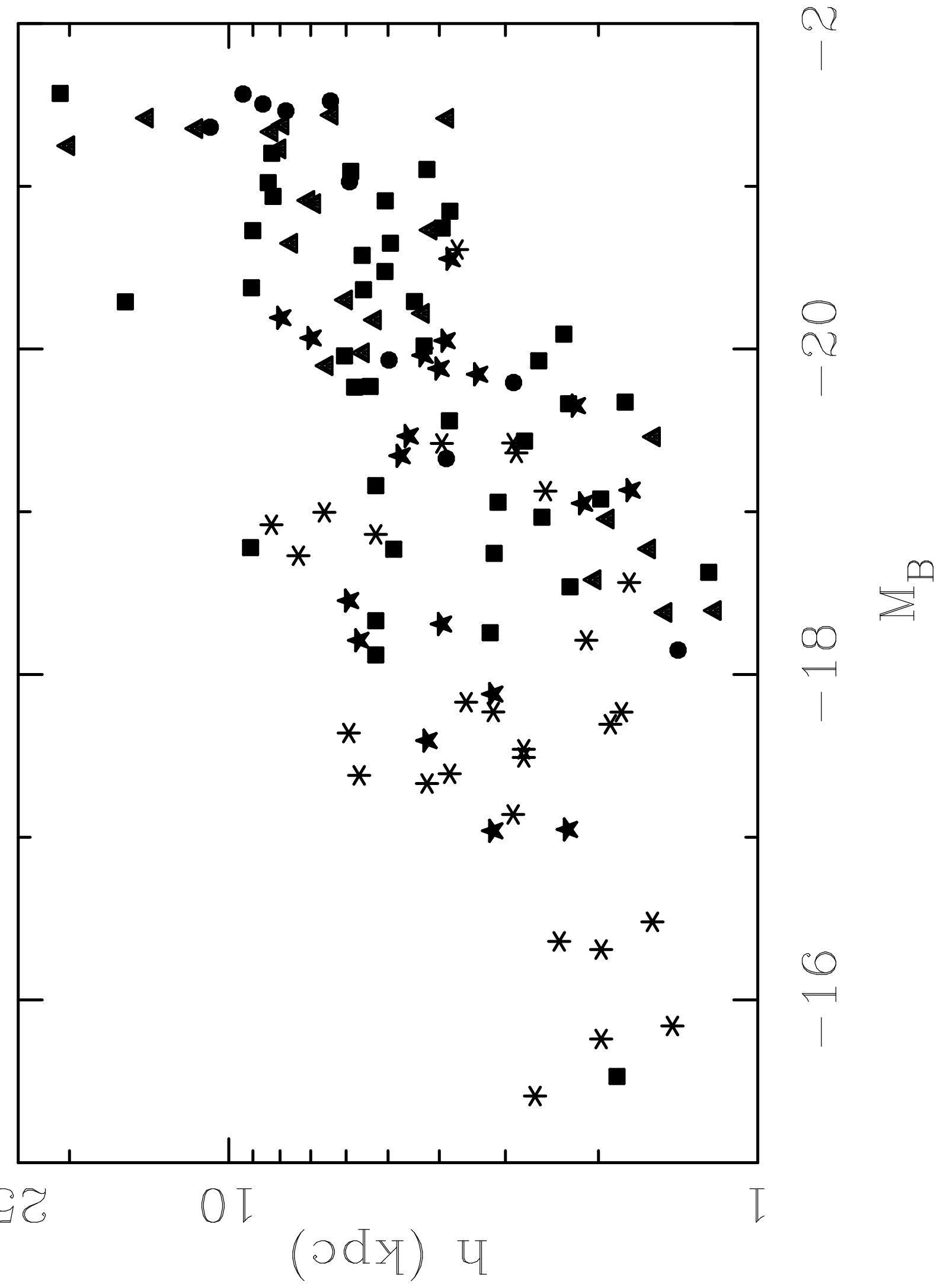




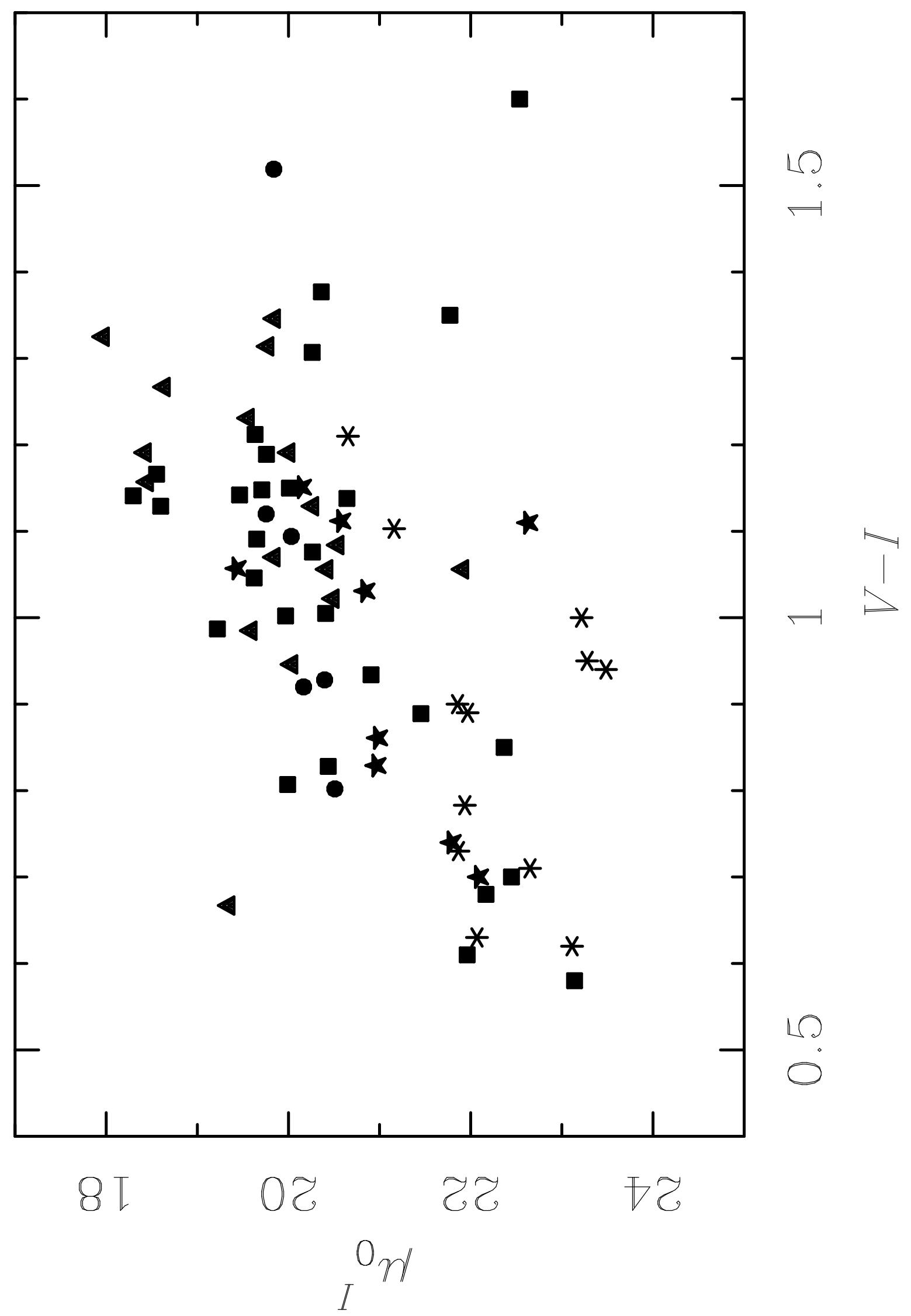




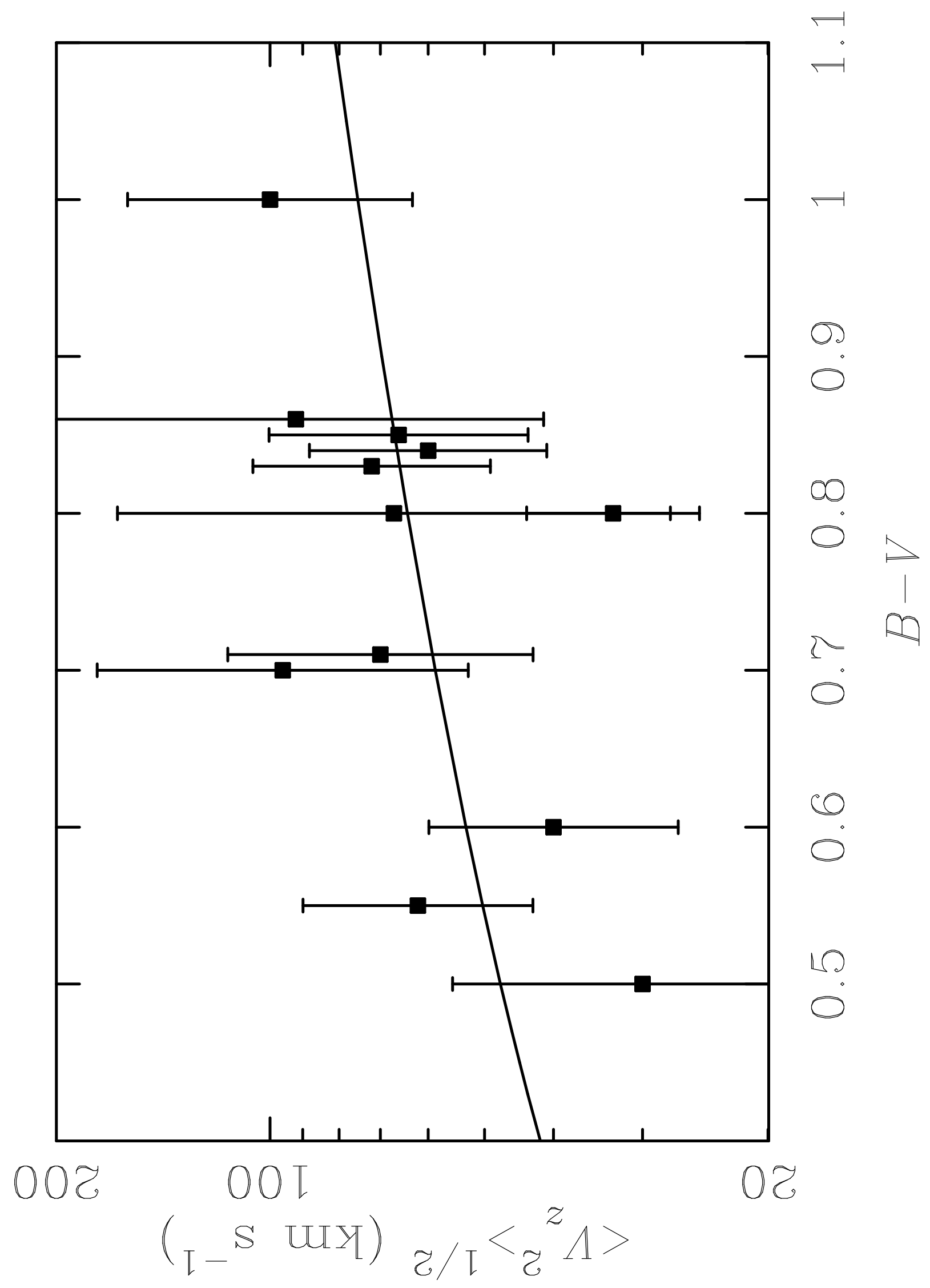




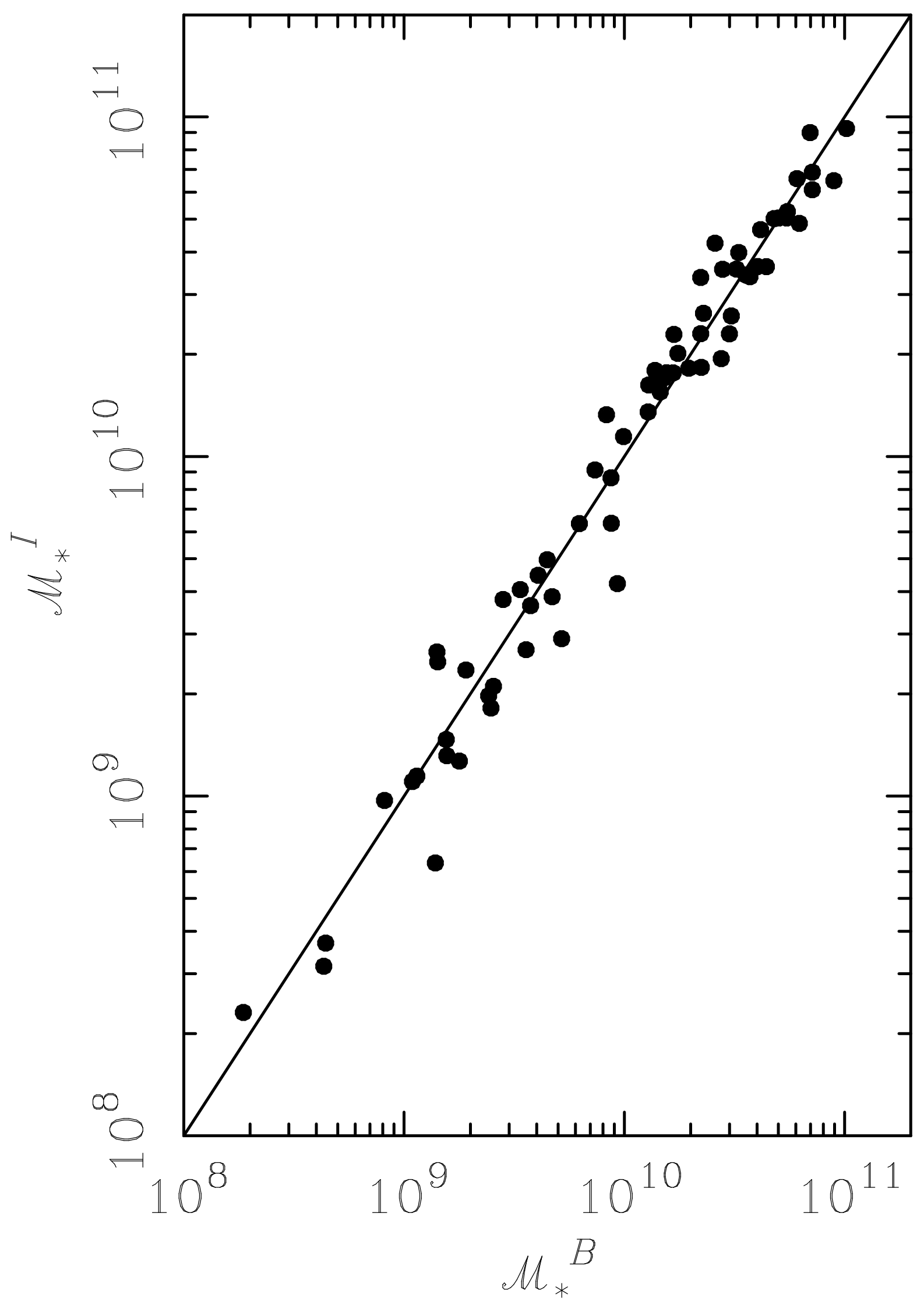




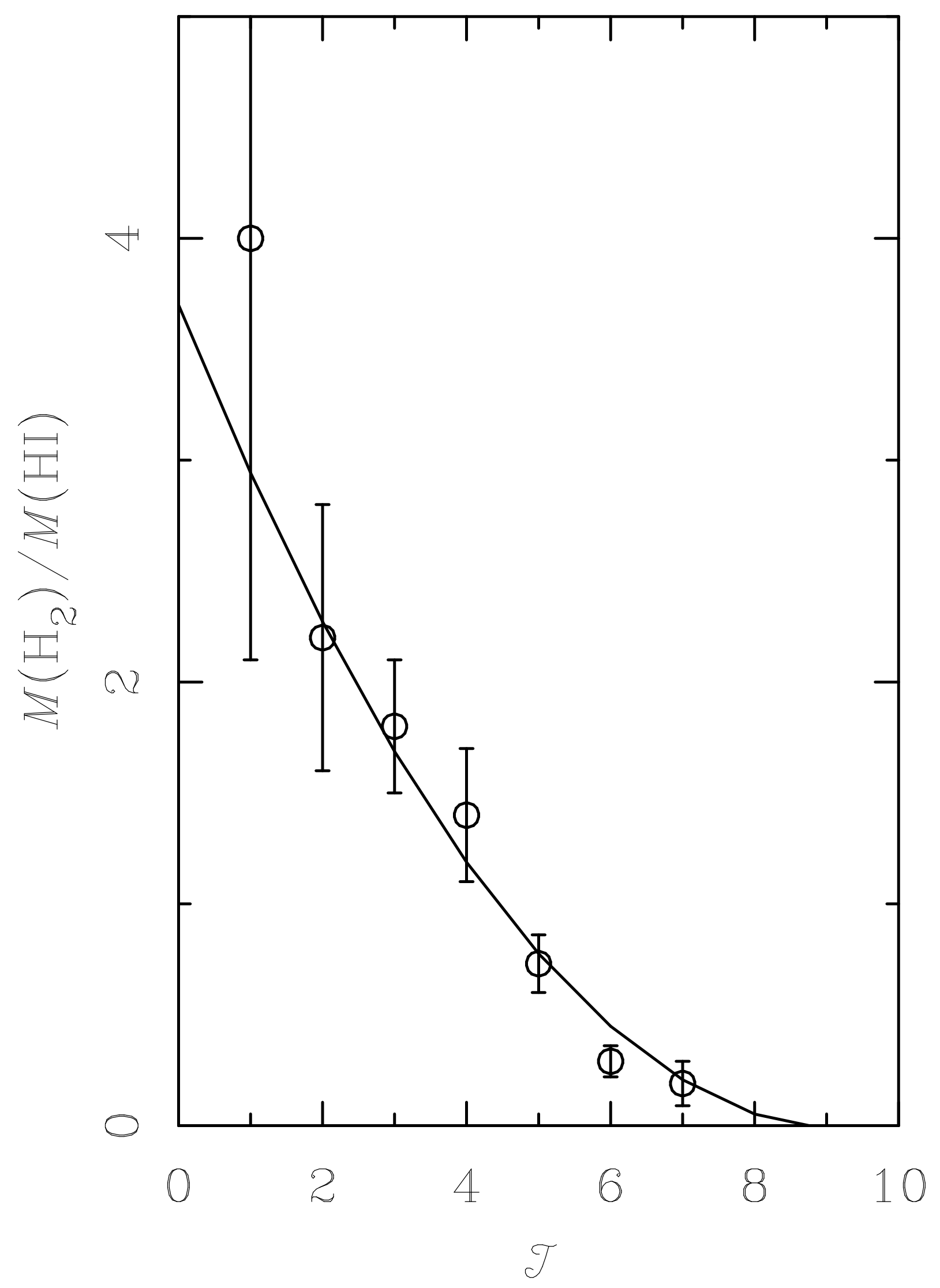




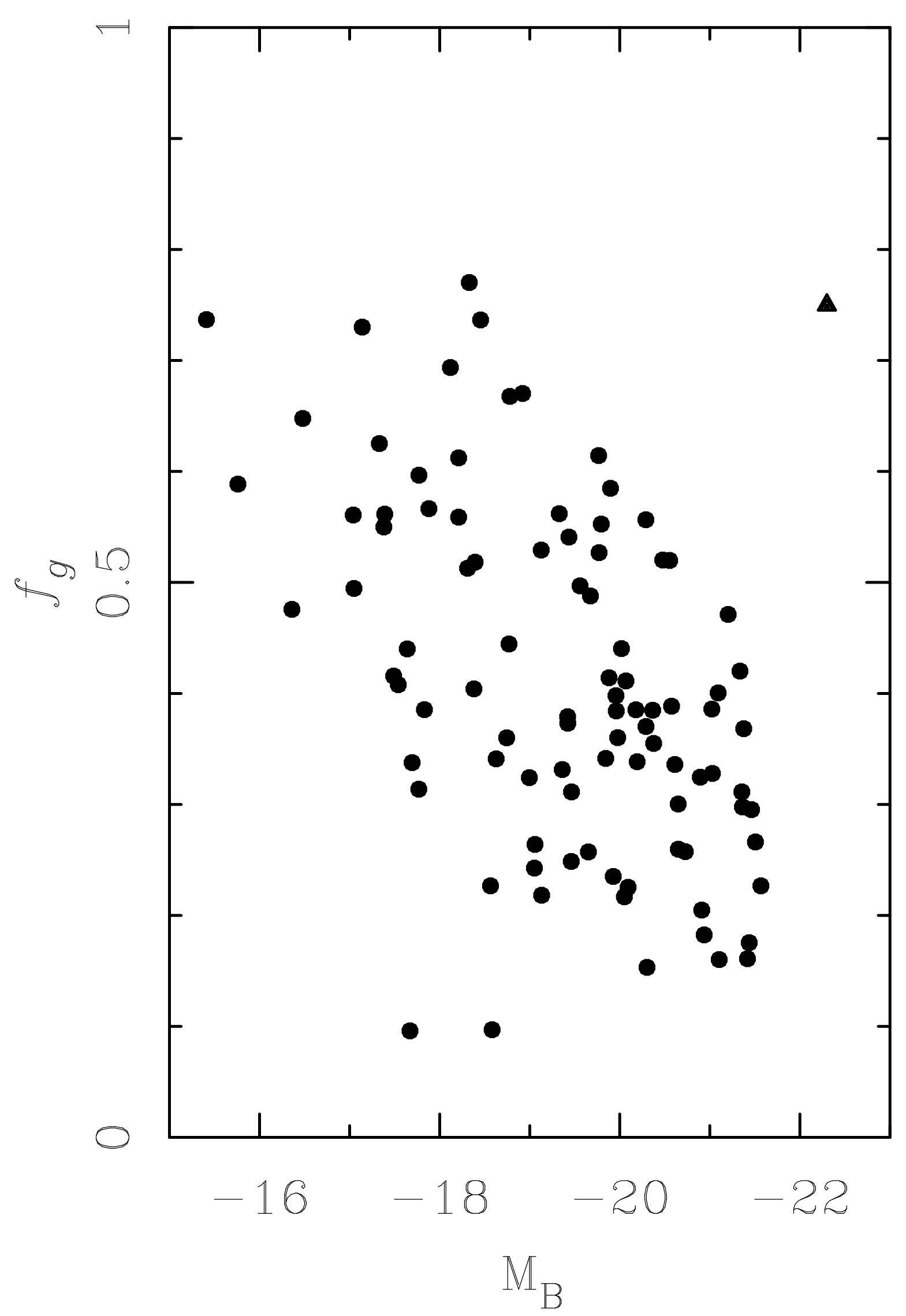




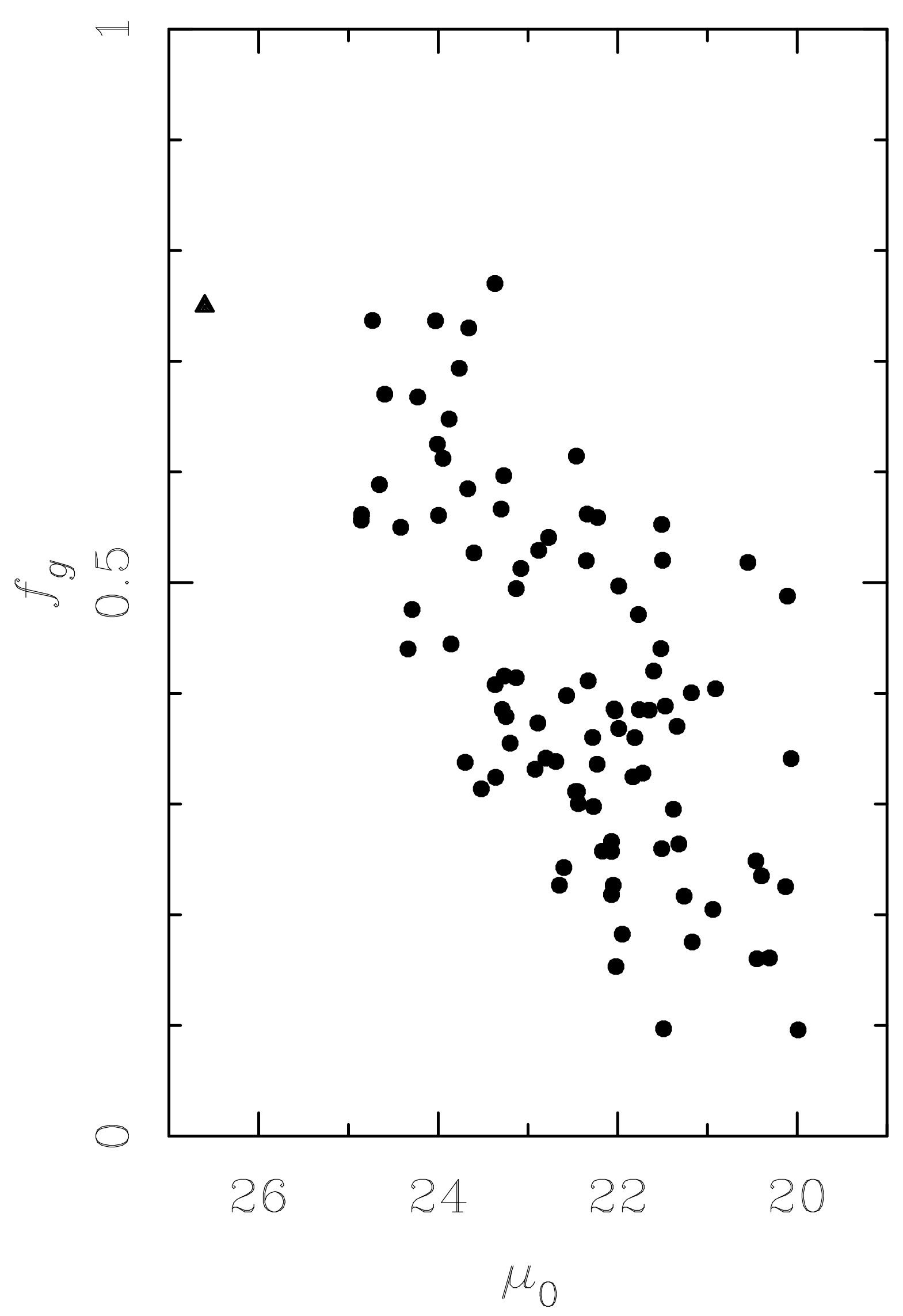




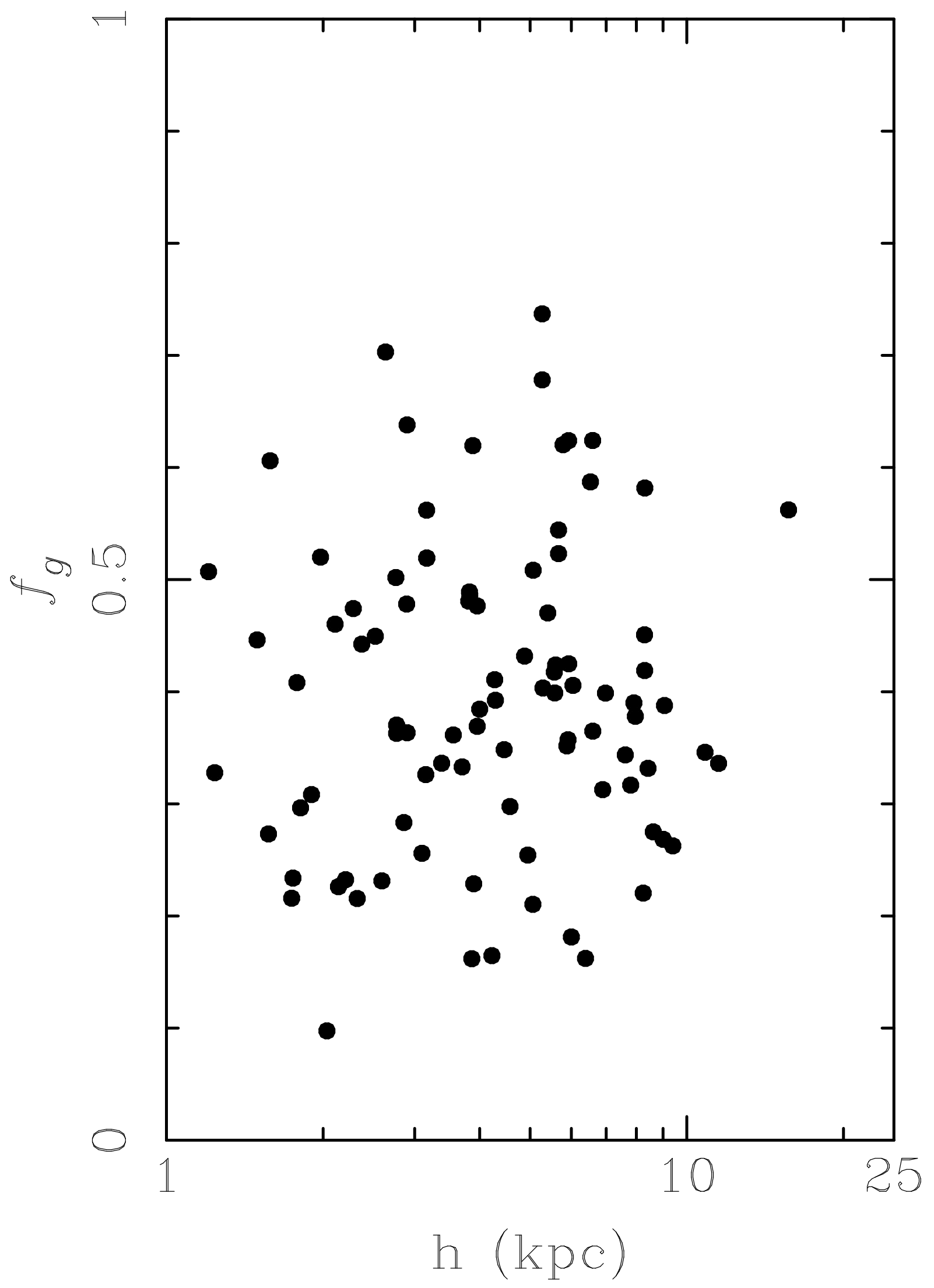




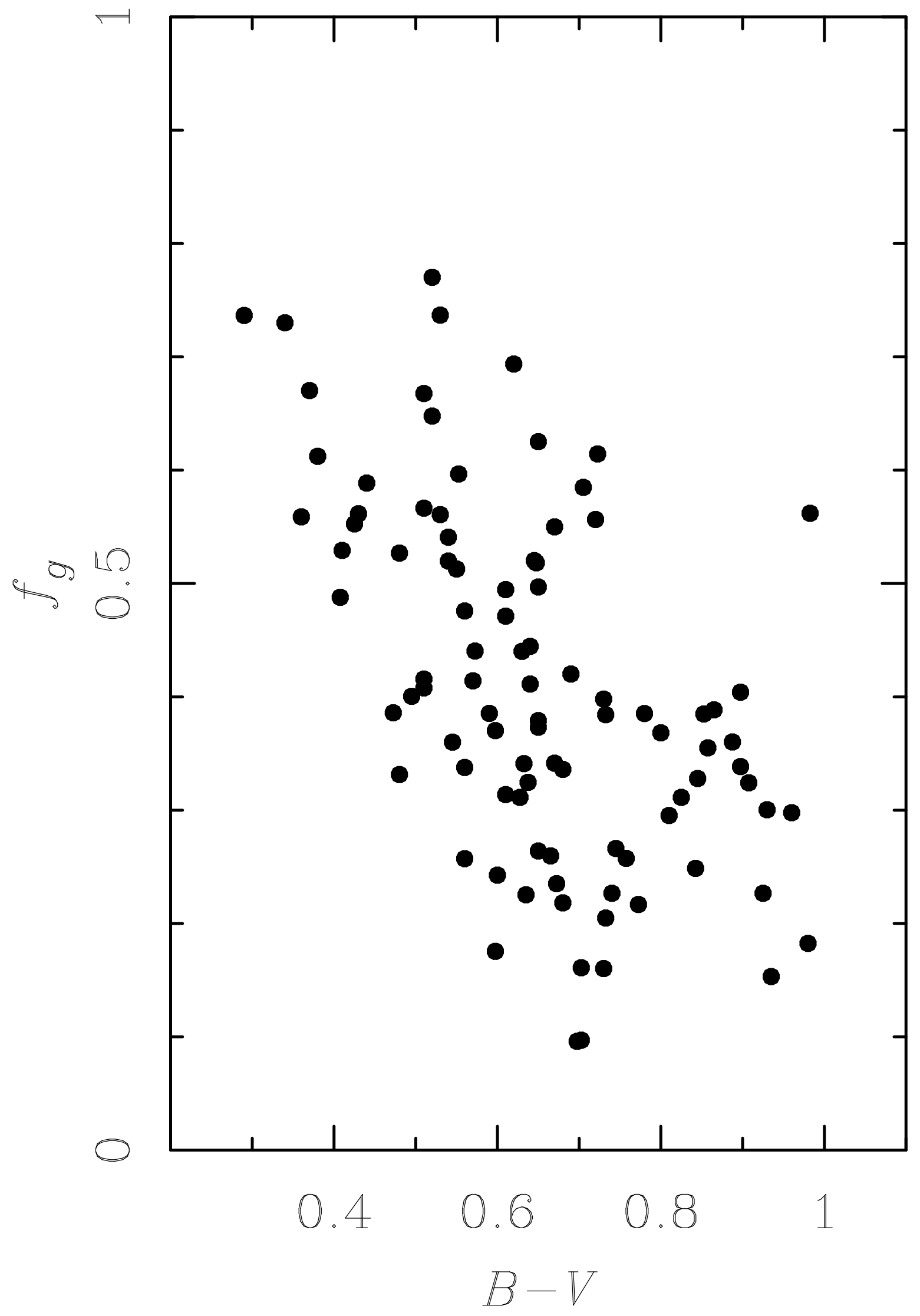




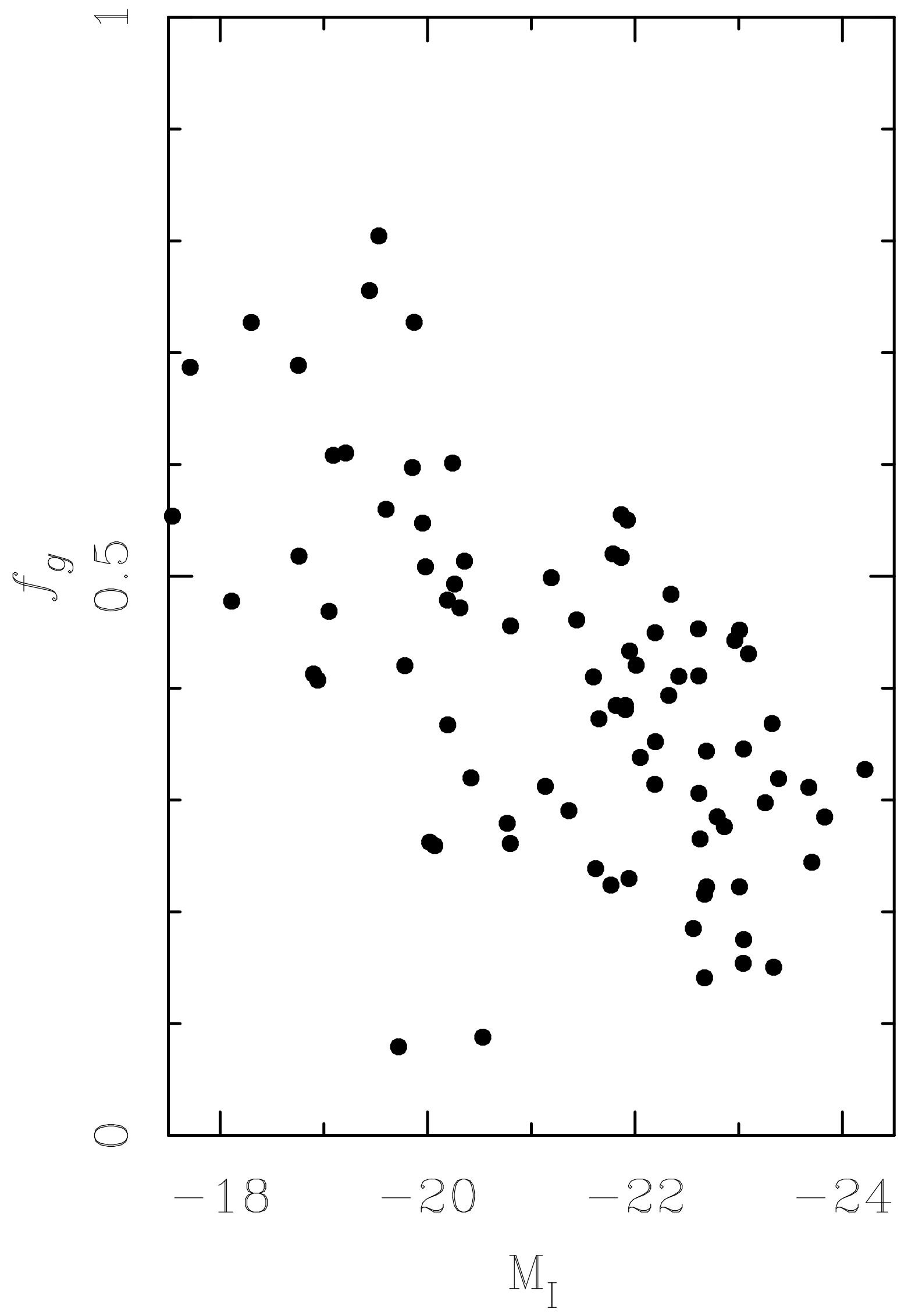




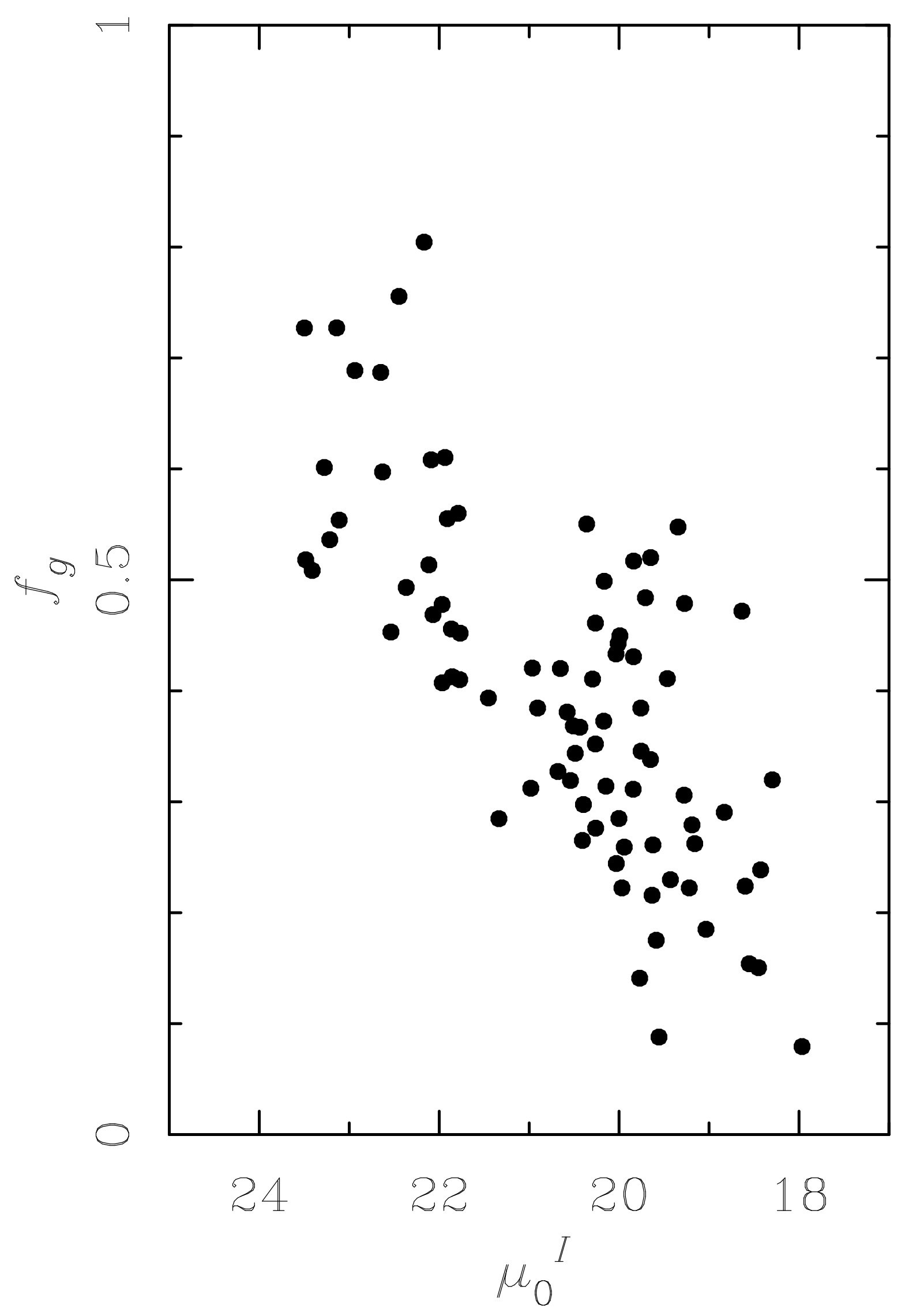




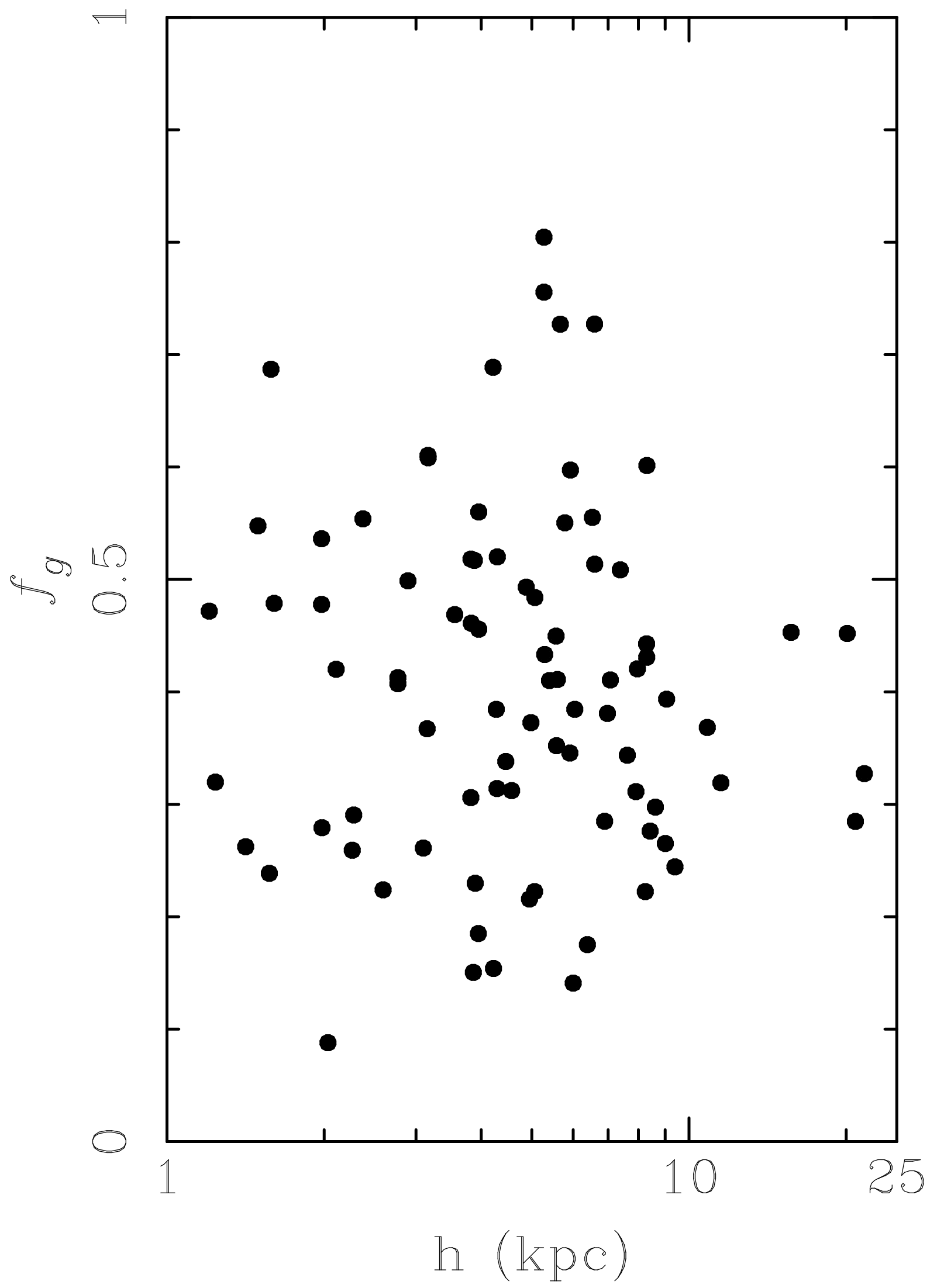




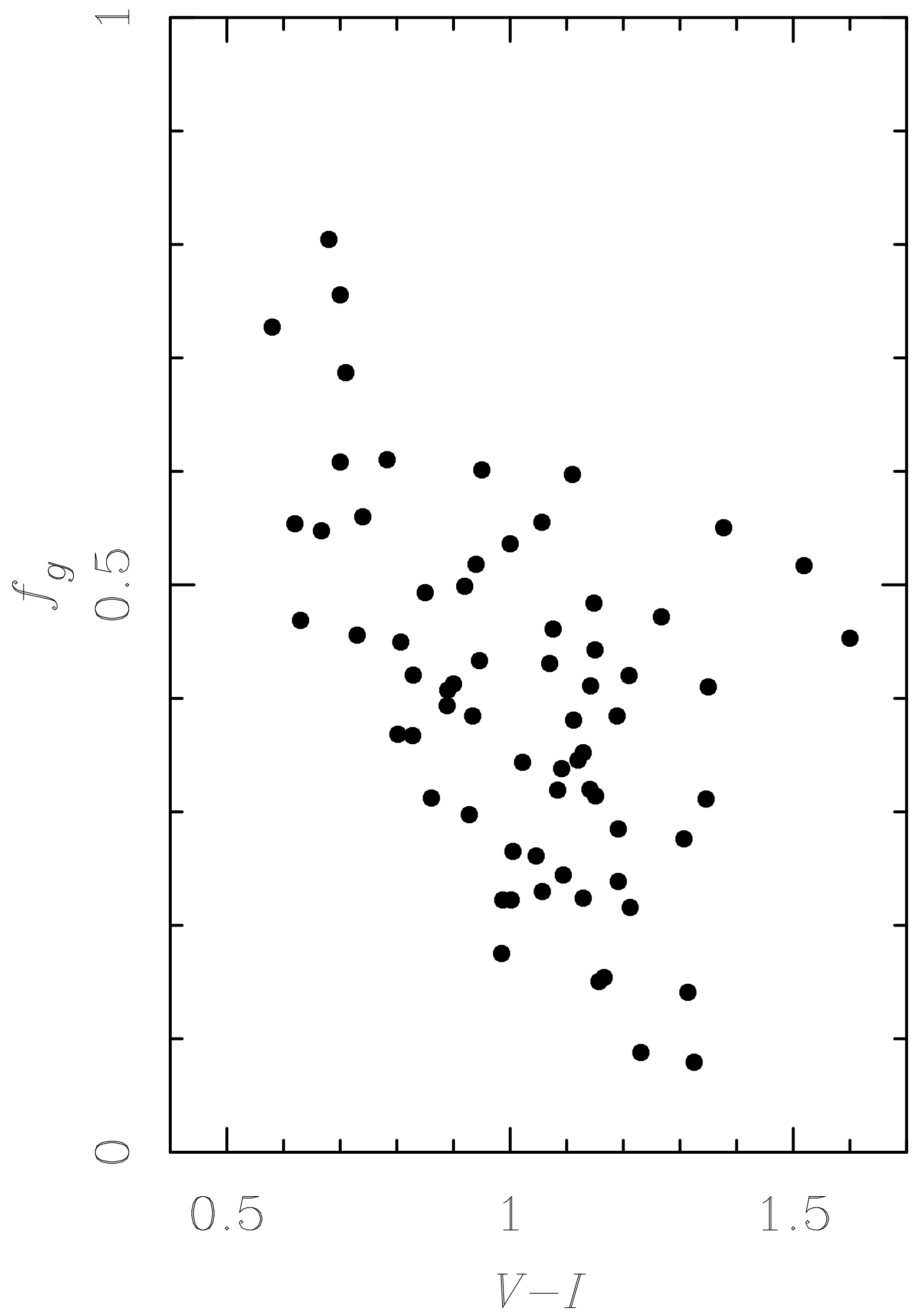




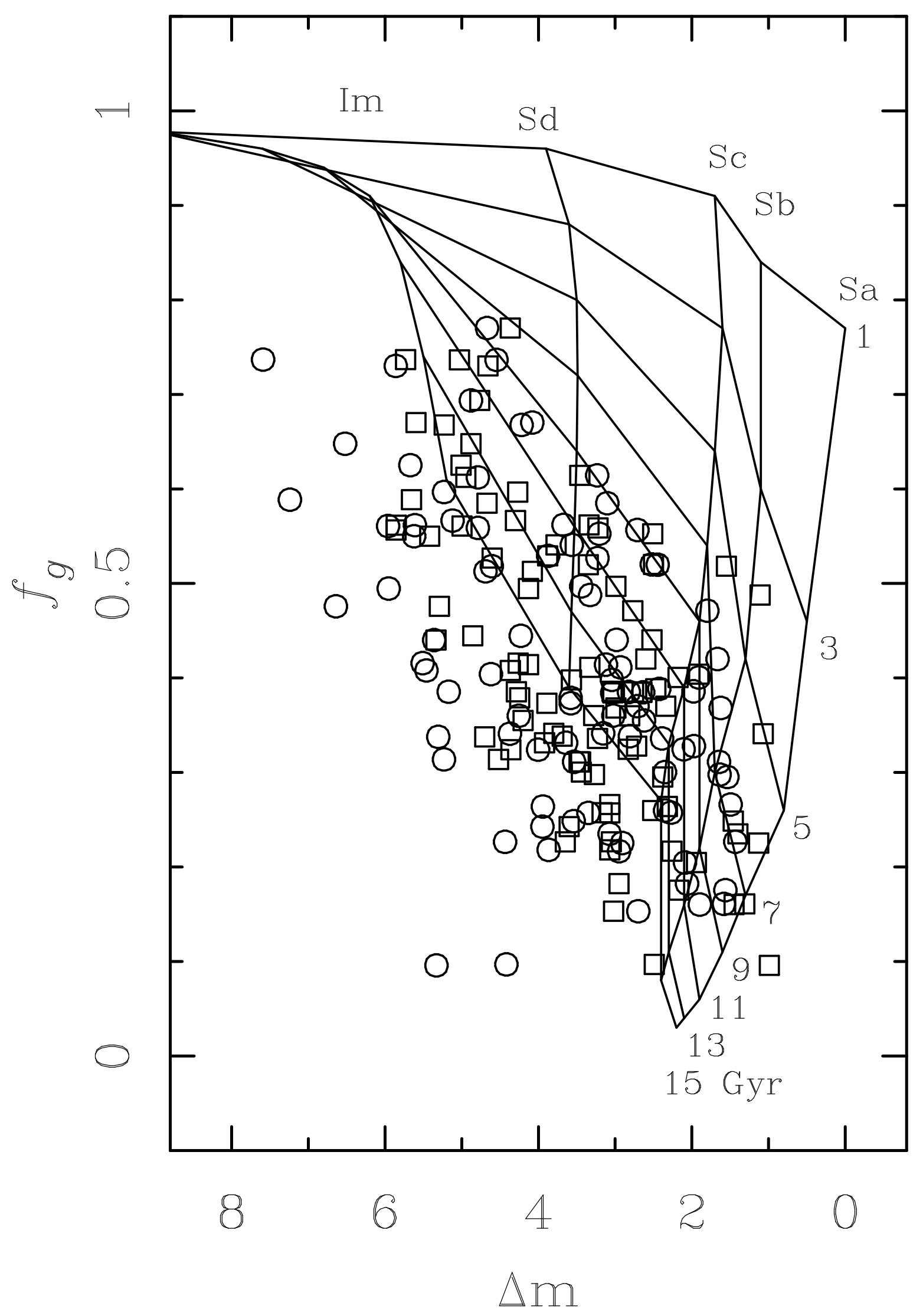

